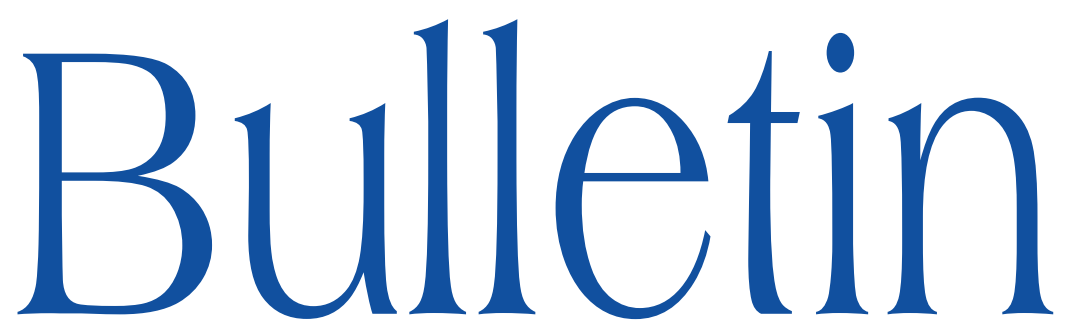

de la SOCIÉTÉ MATHÉMATIQUE DE FRANCE

\title{
LINEARIZATION OF GERMS: REGULAR DEPENDENCE ON THE MULTIPLIER
}

\author{
Stefano Marmi \& Carlo Carminati
}

\section{Tome 136}

Fascicule 4

2008 


\title{
LINEARIZATION OF GERMS: REGULAR DEPENDENCE ON THE MULTIPLIER
}

\author{
By Stefano Marmi \& Carlo Carminati
}

\begin{abstract}
We prove that the linearization of a germ of holomorphic map of the type $F_{\lambda}(z)=\lambda\left(z+O\left(z^{2}\right)\right)$ has a $\mathcal{C}^{1}$-holomorphic dependence on the multiplier $\lambda$. $\mathcal{C}^{1}$ holomorphic functions are $\mathcal{C}^{1}$-Whitney smooth functions, defined on compact subsets and which belong to the kernel of the $\bar{\partial}$ operator.

The linearization is analytic for $|\lambda| \neq 1$ and the unit circle $\mathbb{S}^{1}$ appears as a natural boundary (because of resonances, i.e. roots of unity). However the linearization is still defined at most points of $\mathbb{S}^{1}$, namely those points which lie "far enough from resonances", i.e. when the multiplier satisfies a suitable arithmetical condition. We construct an increasing sequence of compacts which avoid resonances and prove that the linearization belongs to the associated spaces of $\mathcal{C}^{1}$-holomorphic functions. This is a special case of Borel's theory of uniform monogenic functions [2], and the corresponding function space is arcwise-quasianalytic [11]. Among the consequences of these results, we can prove that the linearization admits an asymptotic expansion w.r.t. the multiplier at all points of the unit circle verifying the Brjuno condition: in fact the asymptotic expansion is of Gevrey type at diophantine points.
\end{abstract}

Texte recu le 28 juin 2007, révisé et accepté le 27 février 2008

Stefano Marmi, Scuola Normale Superiore, Piazza dei Cavalieri 7, 56126 Pisa, Italy

E-mail : s.marmi@sns.it

Carlo Carminati, Dipartimento di Matematica, Universita' di Pisa, Largo Bruno Pontecorvo 5, 56127 Pisa, Italy • E-mail : carminat@dm.unipi.it

2000 Mathematics Subject Classification. — 37F50 ; 37F25.

Key words and phrases. - Small divisors, linearization, monogenic functions, quasianalytic space, asymptotic expansion, diophantine condition. 
RÉSumÉ (Linéarisation des germes : dépendence régulière du multiplicateur)

On montre que la linéarisation d'un germe d'application holomorphe du type $F_{\lambda}(z)=\lambda\left(z+O\left(z^{2}\right)\right)$ a une dépendence $\mathcal{C}^{1}$-holomorphe du multiplicateur $\lambda$. Les fonctions $\mathcal{C}^{1}$-holomorphes sont $\mathcal{C}^{1}$ au sens de Whitney, elles sont définies sur des compacts et elles appartiennent au noyau de l'operateur $\bar{\partial}$.

La linéarisation est analytique pour $|\lambda| \neq 1$ et le circle $\mathbb{S}^{1}$ est sa frontière naturelle (due aux résonances, c'est-à-dire les racines de l'unité). Neamoins la linéarisation est encore définie dans la plupart des points de $\mathbb{S}^{1}$, plus précisement aux points qui se trouvent «assez loin des résonances »' et qui correspondent à des conditions arithmétiques adéquates imposées au multiplicateur. Nous construisons une suite croissante d'ensembles compacts qui évitent les résonances et nous démontrons que la linéarisation appartient aux espaces associés aux fonctions $\mathcal{C}^{1}$-holomorphes. C'est un cas particulier de la théorie des fonctions monogènes uniformes de Borel [2], et les espaces de fonctions correspondants sont quasi-analytiques par chemins [11]. Comme conséquence nous montrons que la linéarisation a un développement asymptotique en $\left(\lambda-\lambda_{0}\right)$ dans tous les points $\lambda_{0} \in \mathbb{S}^{1}$ qui verifient la condition de Brjuno. En effet le developpement est du type Gevrey aux points diophantiens.

\section{Introduction}

A germ of holomorphic diffeomorphism of $(\mathbb{C}, 0)$

$$
F_{\lambda}(z)=\lambda\left(z+\sum_{k=2}^{+\infty} f_{k} z^{k}\right), \quad\left(\lambda \in \mathbb{C}^{*}\right)
$$

is linearizable if there exists a holomorphic germ tangent to the identity $H_{\lambda}(z)=$ $z+\sum_{2}^{+\infty} h_{k}(\lambda) z^{k}$ which conjugates $F_{\lambda}$ to the rotation $R_{\lambda}: z \mapsto \lambda z$ namely

$$
F_{\lambda} \circ H_{\lambda}=H_{\lambda} \circ R_{\lambda} .
$$

The derivative $\lambda$ of $F_{\lambda}$ at the fixed point $z=0$ is called the multiplier of $F_{\lambda}$.

If $\lambda$ is not a root of unity there exists a unique formal solution to the conjugacy equation with coefficients $h_{k}, k \geq 2$, determined by the recurrence relation

$$
h_{k}=\frac{1}{\lambda^{k-1}-1} \sum_{j=2}^{k} f_{j} \sum_{\substack{\epsilon \in\left(\mathbb{Z}_{+}\right)^{j} \\|\epsilon|=k}} h_{\epsilon_{1}} \cdots h_{\epsilon_{j}},
$$

where we follow the usual multi-index notation $|\epsilon|=\sum_{i=1}^{j} \epsilon_{i}$. Note that $h_{k} \in \mathbb{C}(\lambda)\left[f_{2}, \ldots, f_{k}\right]$. When $|\lambda| \neq 1 F_{\lambda}$ is always linearizable (by the classical Koenigs-Poincaré theorem); nevertheless the classical estimates on radius of convergence of $H_{\lambda}$ deteriorate as $|\lambda| \rightarrow 1$. In the elliptic case, i.e. when $\lambda=e^{2 \pi i \alpha}$ and $\alpha \in \mathbb{R} \backslash \mathbb{Q}$, the linearization need not be convergent due to the contribution from small denominators in (3). After the work of Brjuno [3] and Yoccoz [18] we know that all holomorphic germs with multiplier 
$\lambda=e^{2 \pi i \alpha}$ are analytically linearizable if and only if $\alpha$ verifies the Brjuno condition $\mathcal{B}(\alpha)<+\infty$, where $\mathcal{B}$ is the Brjuno function (see the next Section for its definition and properties).

Let us normalize $F_{\lambda}$ asking that it is defined and univalent on the unit disk $\mathbb{D}$. Then one can prove directly, using the classical majorant series' method and Davie's Lemma (see [5] [4]), that there are positive constants $b_{0}, c_{0}$ (that do not depend on $\alpha$ ) such that

$$
\left|h_{k}\right| \leq c_{0} e^{k\left(\mathcal{B}(\alpha)+b_{0}\right)}
$$

where $\lambda=e^{2 \pi i \alpha}, \alpha \in \mathbb{R}$ and $\mathcal{B}$ is the Brjuno function ${ }^{(1)}$.

The same estimate (4) (with larger values $b_{0}$ and $c_{0}$ ) holds uniformly with respect to $\lambda^{\prime}$ in a cone with vertex in $e^{2 \pi i \alpha}$. Therefore for any $\varepsilon>0$ we will be able to define a closed set $C$ such that there exists $\rho>0$ such that

(i) $\operatorname{meas}_{2}(\mathbb{C} \backslash C) \leq \varepsilon$ and $\operatorname{meas}_{1}\left(C \cap \mathbb{S}^{1}\right) \geq 2 \pi-\varepsilon$,

(ii) for each $\lambda \in C$ the linearization $H_{\lambda}$ is holomorphic and bounded on $\mathbb{D}_{\rho}=\{z \in \mathbb{C}:|z| \leq \rho\}$.

(Here meas $_{d}, d \in\{1,2\}$ denotes the $d$-dimensional Lebesgue measure).

The construction of such a set is performed removing from $\mathbb{C}$ the union of suitably small connected open neighbourhoods around the roots of unity ${ }^{(2)}$ and its detailed description can be found in Section 3.1; it will be evident from the construction that the radius $\rho$ tends to 0 as $\epsilon$ tends to 0 .

Let us point out that the property (ii) above means that a uniform lower bound on the radius of convergence of $H_{\lambda}$ holds as $\lambda$ varies in $C$, even near the unit circle. The set $C$ is sort of a "bridge" joining the two connected components of the set of parameter values considered in the Koenigs-Poincare theorem, crossing the unit circle at some values $\lambda=e^{2 \pi i \alpha}$ with $\alpha$ a Brjuno number.

We address the problem of studying the regularity of this map $\lambda \mapsto H_{\lambda}$ : we will prove global regularity results (see Theorem A below) and local regularity results (Theorem B).

The global regularity results we prove are inspired by the work of Borel on uniform monogenic functions [2]. Borel extended the notion of holomorphic function so as to include functions defined on closed subsets of $\mathbb{C}$. His uniform monogenic functions (whose precise definition we recall and recast in modern terminology in Appendix B) can have, in certain situations, analytic

(1) It is known that there are different objects that are called "Brjuno function"; nevertheless for this estimate is quite irrelevant which one we choose, since the difference of two Brjuno functions is bounded by a universal constant, i.e. independent of $\alpha$ (see Section 2).

(2) The property (i) can be realized just asking that the "size" of the neighbourhood of each root decays sufficently fast when the order of the root increases. 
continuation through what is considered as a natural boundary of analyticity in Weierstrass' theory. One of Borel's goals was to determine, with the help of Cauchy's formula, sufficiently general conditions which would have ensured uniqueness of the monogenic continuation, i.e. a quasianalyticity property (see [15], [17] for a modern discussion of this part).

The importance of Borel's monogenic functions in parameter-dependent small divisor problems was emphasized by Kolmogorov [9]. Arnol'd discussed in detail this issue in his work [1] on the local linearization problem of analytic diffeomorphisms of the circle (see [19] for a very nice introduction and for the most complete results on the subject). Arnol'd complexified the rotation number but he did not prove that the dependence of the conjugacy on it is monogenic. This point was dealt with by M. Herman [7] who also reformulated Borel's ideas using the modern terminology, Whitney's theory [16] on differentiability of functions on closed sets and the theory of uniform algebras of (analytic) functions defined on closed sets in the complex plane. It is Herman's point of view which was developed in [10] and which we will summarize in Appendix B, where we recall the formal definition of $\mathcal{C}^{1}$-holomorphic and $\mathcal{C}^{\infty}$-holomorphic functions. Later Risler [13] extended considerably part of Herman's work proving various regularity results under less restrictive arithmetical conditions, namely using the Brjuno conditon as in [19] instead of a more classical diophantine condition. One should also mention that Whitney smooth dependence on parameters has been established also in the more general framework of KAM theory by Pöschel [12] who did not however consider neither complex frequencies nor Brjuno numbers.

In this paper we will extend the results of Herman and Riesler to the case of germs of holomorphic diffeomorphisms of $(\mathbb{C}, 0)$. Our proofs will in fact be more elementary since in this case one can use a direct approach and the majorant series method applies (see, e.g. [4]).

Let us point out that, although the linearization problem makes no sense for $\lambda=0$, nevertheless the recurrence (3) defines a function $H: \lambda \mapsto H_{\lambda}$ which turns out to be well defined and holomorphic at the origin: if we denote with $\mathcal{F}_{0}=z+\sum_{k=2}^{+\infty} f_{k} z^{k}$, so that $F_{\lambda}=\lambda \mathcal{F}_{0}$, then $H_{0}$ turns out to be simply the inverse of $\mathcal{F}_{0}: \mathcal{F}_{0}\left(H_{0}(z)\right)=z$. In fact $H$ can even be extended analitically at infinity just setting $H_{\infty}(z)=z$. Therefore we may consider $H$ as defined on $C \cup\{\infty\}$ which is a compact subset of $\mathbb{P}^{1} \mathbb{C}$ : this has an important consequence since it is proved in [11] that the space of $\mathcal{C}^{1}$-holomorphic functions to which $H$ belongs (see Theorem A below) is arcwise quasianalytic ${ }^{(3)}$.

(3) A function space $X$ is said to be arcwise quasianalytic iff the only function that belongs to $X$ and vanishes on an arbitrarily short arc is the null function.

TOME $136-2008-\mathrm{N}^{\mathrm{O}} 4$ 
Let us state the main results. In what follows we will assume the germ $F_{\lambda}$ to be of the form (1), defined and univalent on the unit disk $\mathbb{D}$. For any $\rho>0$, $\mathbb{D}_{\rho}:=\{z \in \mathbb{C}:|z| \leq \rho\}$ and $\mathcal{H}^{\infty}\left(\mathbb{D}_{\rho}\right)$ will denote the complex Banach space of functions holomorphic and bounded on $\mathbb{D}_{\rho}$.

Theorem A (Global Regularity). - For any $\varepsilon>0$ there exist $\rho>0$ and two connected closed sets $C^{*}$ and $C$ such that

(a) $C^{*} \subset C \subset \mathbb{P}^{1} \mathbb{C}, \operatorname{meas}_{2}\left(\mathbb{C} \backslash C^{*}\right) \leq \varepsilon$ and $\operatorname{meas}_{1}\left(C \cap \mathbb{S}^{1}\right) \geq 2 \pi-\varepsilon$

(b) $H \in C_{\text {hol }}^{1}\left(C, \mathcal{H}^{\infty}\left(\mathbb{D}_{\rho}\right)\right)$

(b*) $H \in C_{\mathrm{hol}}^{\infty}\left(C^{*}, \mathcal{H}^{\infty}\left(\mathbb{D}_{\rho}\right)\right)$

As will be evident in Section 4.1, for any fixed value $\bar{\lambda}=e^{2 \pi i \bar{\alpha}}$ on the unit circle with $\mathcal{B}(\bar{\alpha})<+\infty$ we can manage to build $C^{*}$ so that $\bar{\lambda} \in C^{*}$. Therefore Theorem A proves that, by a suitable chioice of the set $C^{*}$, one can extend $H$ and all its derivatives at any Brjuno point on the circle and this leads to the existence of asymptotic expansions for the linearization $H$ at Brjuno points.

In fact we can prove that this expansion is quite regular at diophantine points:

Theorem B (Local Regularity). - If $\alpha_{0}$ is a diophantine point ${ }^{(4)}$ with exponent $\tau_{0} \geq 2$ and $\lambda_{0}=e^{2 \pi i \alpha_{0}}$, there exists $\rho>0$ such that for any pair of disks $\Delta^{-} \subset \mathbb{D}$ and $\Delta^{+} \subset \mathbb{C} \backslash \mathbb{D}$ tangent to $\mathbb{S}^{1}$ in $\lambda_{0}$ the map

$$
\overline{\Delta^{+} \cup \Delta^{-}} \ni \lambda \mapsto H_{\lambda} \in \mathcal{H}^{\infty}\left(\mathbb{D}_{\rho}\right)
$$

has a Gevrey- $\tau_{0}$ asymptotic expansion in $\lambda_{0}$ (we refer the reader to the beginning of Section 5 for its precise definition, see especially (30)).

We briefly summarize the content of the paper. In Section 2 we define the Brjuno series $\mathcal{B}$ and we prove several properties of its sublevel sets. Since $\mathcal{B}$ is lower semicontinuous it follows that the complement of any given sublevel set $\{x \in \mathbb{R}, \mathcal{B}(x) \leq t\}$ is the countable disjoint union of open intervals. These intervals are "centered" at those rational values for which the finite version of the Brjuno series is bounded by the value $t$ defining the sublevel set considered. The discussion of Section 2 prepares the ground for the definition of the domain $C$ where the conjugation $H: \lambda \mapsto H_{\lambda}$ is $\mathcal{C}^{1}$-holomorphic. The proof of the $C_{\mathrm{hol}^{-}}^{1}$ regularity of $H$ is the main result of Section 3 while in Section 4 we shall restrict the domain of $H$ to a suitably chosen set $C^{*} \subset C$ to gain $C_{\text {hol }}^{\infty}$-regularity of the conjugation. The proof of Theorem A can be easily obtained gathering the results of Section 3 (Theorem 1) and Section 4 (Theorem 2). In Section 5

(4) Let us recall that an irrational number $\alpha_{0}$ is diophantine with exponent $\tau_{0} \geq 2$ if and only if there exists $\gamma>0$ such that for all $p / q \in \mathbb{Q}$ one has $\left|\alpha_{0}-p / q\right| \geq \gamma q^{-\tau_{0}}$. 
we introduce other arithmetically defined real Cantor sets which are useful to establish the Gevrey regularity of $H$ as claimed in Theorem B.

In the two appendices we recall some elementary properties of the continued fraction expansion of a real number (Appendix A) and the definition of $\mathcal{C}^{1}$ holomorphic, $\mathcal{C}^{\infty}$-holomorphic and monogenic functions (Appendix B).

\section{Geometry of the sublevel sets of the Brjuno function}

In the following we shall always denote with $\alpha$ an irrational number; its continued fraction expansion will be denoted $\alpha=\left[a_{0}, a_{1}, a_{2}, \ldots, a_{N}, \ldots\right]$ where $a_{0} \in \mathbb{Z}$ and $a_{j} \in \mathbb{Z}_{+}, j \geq 1$, are the partial quotients and $p_{j} / q_{j}=\left[a_{0}, a_{1}, a_{2}, \ldots, a_{j}\right]$ is the $j$-th convergent of $\alpha$. If $a_{0} \in \mathbb{Z}$ and $\left(a_{1}, \ldots, a_{N}\right) \in\left(\mathbb{Z}_{+}\right)^{N}$ we denote $I\left(a_{0}, \ldots a_{N}\right)$ the set of real number whose continued fraction begins with the string $\left(a_{0}, \ldots, a_{N}\right)$. This set is in fact an interval and it is usually called the cylinder associated to the string of symbols $\left(a_{0}, \ldots, a_{N}\right)$ (for more details and classical results about continued fraction expansions we refer to appendix A).

To construct the domains on which we will prove the regularity of the conjugation we shall use the 1-periodic function $\mathcal{B}$ defined by the following Brjuno series

$$
\mathcal{B}(\alpha):=\sum_{k=0}^{+\infty} \frac{\log a_{k+1}}{q_{k}} \quad(\alpha \in \mathbb{R} \backslash \mathbb{Q}), \quad \mathcal{B}(r)=+\infty \text { if } r \in \mathbb{Q},
$$

which is closely related to the classical Brjuno series (see [3])

$$
\mathcal{B}_{c l}(\alpha):=\sum_{k=0}^{+\infty} \frac{\log q_{k+1}}{q_{k}}
$$

In fact it is easily seen that

$$
0 \leq \mathcal{B}_{c l}(\alpha)-\mathcal{B}(\alpha) \leq \sum_{k=0}^{+\infty} \frac{\log \left(2 F_{k}\right)}{F_{k}}<+\infty,
$$

(where $F_{k}$ are the Fibonacci numbers).

The above inequalities show that the bound (4) on the growth of the coefficents of the linearization, which holds for the classical Brjuno function, must be valid for the Brjuno function $\mathcal{B}$ as well (possibly choosing a larger value for the universal constant $b_{0}$ ). We have chosen $\mathcal{B}$ instead of $\mathcal{B}_{c l}$ because it has various nice properties: its global minimum is 0 and is attained at the golden mean $\phi_{0}:=\frac{\sqrt{5}-1}{2}$. Moreover the set $\Phi$ of all local minima of $\mathcal{B}$ is just the set 
of preimages of the golden mean relative to the Gauss map $\mathcal{G}(x)=\{1 / x\}$ (see also Lemma 2 below):

$$
\Phi:=\left\{\phi \in \mathbb{R}: \mathcal{G}^{(n)}(\phi)=\phi_{0} \text { for some } n \in \mathbb{N}\right\} .
$$

Therefore to any element $\phi \in \Phi$ corresponds a continued fraction expansion of the form

$$
\phi=\left[a_{0}, \ldots, a_{N}, 1,1,1, \ldots\right]=\left[a_{0}, \ldots, a_{N}+\phi_{0}\right] .
$$

In the sequel we will always use only $\mathcal{B}$. The following lemmata will be useful to give a neat description of sub/super-level sets of the function $\mathcal{B}$.

Lemma 1. - If $x \in \mathbb{R} \backslash \mathbb{Q}$ and $\mathcal{B}(x)<+\infty$ then for all $\varepsilon>0$ exists $\alpha^{ \pm}$such that

(i) $\alpha^{-}<x<\alpha^{+}$and $\alpha^{+}-\alpha^{-}<\varepsilon$,

(ii) $\left|\mathcal{B}\left(\alpha^{ \pm}\right)-\mathcal{B}(x)\right|<\varepsilon$.

Proof. - Let $x:=\left[a_{0}, \ldots, a_{N-1}, a_{N}, a_{N+1}, \ldots\right]$; fix $N$ odd and such that

$$
\frac{1}{F_{N}}<\varepsilon, \quad \sum_{k=N}^{+\infty} \frac{\log a_{k+1}}{q_{k}}<\varepsilon,
$$

and set

$$
\alpha^{+}:=\left[a_{0}, \ldots, a_{N-1}, 2 a_{N}+\phi_{0}\right], \quad \alpha^{-}:=\left[a_{0}, \ldots, a_{N-1}, a_{N}, 2 a_{N+1}+\phi_{0}\right] .
$$

It is clear that $\alpha^{ \pm} \in I\left(a_{0}, \ldots, a_{N-1}\right)$ hence by (35) in Appendix A

$$
\left|\alpha^{+}-\alpha^{-}\right| \leq\left|\frac{p_{N-1}}{q_{N-1}}-\frac{p_{N-1}+p_{N-2}}{q_{N-1}+q_{N-2}}\right|=\frac{1}{q_{N-1}\left(q_{N-1}+q_{N-2}\right)} \leq \frac{1}{F_{N} F_{N-1}} .
$$

On the other hand

$$
\varepsilon>\frac{\log 2}{F_{N}} \geq \mathcal{B}\left(\alpha^{ \pm}\right)-\mathcal{B}(x) \geq-\sum_{k=N}^{+\infty} \frac{\log a_{k+1}}{q_{k}}>-\varepsilon .
$$

LEMMA 2. - If $x:=\left[a_{0}, \ldots, a_{N-1}, a_{N}, a_{N+1}, \ldots\right]$ is such that $a_{2 n}>1$ for infinitely many $n \in \mathbb{N}$, then for all $\varepsilon>0$ there exists $\alpha^{-}$such that

(i) $x-\varepsilon<\alpha^{-}<x$,

(ii) $\mathcal{B}\left(\alpha^{-}\right)<\mathcal{B}(x)$.

On the other hand if for infinitely many $n \in \mathbb{N} a_{2 n+1}>1$, then for all $\varepsilon>0$ there exists $\alpha^{+}$such that

(i) $x+\varepsilon>\alpha^{+}>x$,

(ii) $\mathcal{B}\left(\alpha^{+}\right)<\mathcal{B}(x)$. 
Proof. - It is enough to choose $n$ big enough and such that $a_{2 n}>1$ and set

$$
\alpha^{-}:=\left[a_{0}, \ldots, a_{2 n-1}, a_{2 n}-1+\phi_{0}\right],
$$

so that $\mathcal{B}(x)-\mathcal{B}\left(\alpha^{-}\right)=-\frac{\log \left(1-a_{2 n}^{-1}\right)}{q_{2 n-1}}+\sum_{k=2 n}^{+\infty} \frac{\log a_{k+1}}{q_{k}}>0$. With a similar trick one can determine $\alpha^{+}$.

Lemma 3. - The function $\mathcal{B}$ is lower semicontinuous.

Proof. - Let $A_{t}:=\{x \in \mathbb{R}: \mathcal{B}(x)>t\},(t \geq 0)$, denote the $t$-superlevel of the function $\mathcal{B}$. To prove that $\mathcal{B}$ is semicontinuous it is enough to show that $A_{t}$ is open for all $t \geq 0$. If $\mathcal{B}(\bar{x})>t$ and $\bar{x}=\left[a_{0}, a_{1}, a_{2}, \ldots, a_{N}, \ldots\right] \in \mathbb{R} \backslash \mathbb{Q}$ then, for some $N \in \mathbb{N}, \sum_{k=0}^{N} \frac{\log a_{k+1}}{q_{k}}>t$. Hence $\mathcal{B}(x)>t$ for all $x \in I\left(a_{0}, \ldots, a_{N+1}\right)$. A simpler argument settles the case $\bar{x}$ is rational.

It is easy to prove that if $V=] \xi^{-}, \xi^{+}$[ is an open interval with irrational endpoints and $\left|\xi^{+}-\xi^{-}\right|<1$ then there is a unique rational point $\bar{p} / \bar{q} \in V$ such that $\bar{q}<q$ for all $p / q \in V \backslash\{\bar{p} / \bar{q}\}$; we will call the rational point $\bar{p} / \bar{q}$ the pseudocenter of the interval $V$. Since $\mathcal{B}$ is lower semicontinuous we know that the complement of each sublevel is the countable union of disjoint open intervals with irrational endpoints. Each of these intervals will be labeled by its pseudocenter.

In order to characterize the set $\mathcal{Q}_{t}$ of pseudocenters of the connected components of the complement of the sublevel $\{\mathcal{B}(x) \leq t\}$ we introduce a finite Brjuno function (simply denoted by $B_{f}$ ) which is defined on finite continued fractions by the formula ${ }^{(5)}$

$$
B_{f}\left(\left[a_{0}, \ldots, a_{n}\right]\right):=\sum_{k=0}^{n-1} \frac{\log a_{k+1}}{q_{k}} .
$$

The following lemma gives an accurate description of each of the countable connected components of the $t$-superlevel sets $A_{t}$ of the Brjuno function $\mathcal{B}$ and a precise characterization of the set $\mathcal{Q}_{t}$.

Lemma 4. - Let $V=] \xi^{-}, \xi^{+}\left[\right.$be a connected component of $A_{t}$ and let

$$
\xi^{ \pm}:=\left[a_{0}, . ., a_{N-1}, a_{N}^{ \pm}, a_{N+1}^{ \pm}, \ldots\right], \quad N \geq 1, \quad a_{N}^{+} \neq a_{N}^{-} .
$$

Then

(i) $\mathcal{B}\left(\xi^{ \pm}\right)=t$;

(5) The choice of defining $B_{f}$ on finite continued fractions instead of $\mathbb{Q}$ avoids the ambiguity which arises from the fact that each rational number admits two different continued fraction expansions, see the Remark below the proof of Lemma 4 .

TOME $136-2008-\mathrm{N}^{\mathrm{O}} 4$ 
(ii) $a_{N}^{+} \geq 2, a_{N}^{-}=a_{N}^{+}-1, a_{N+2 k}^{+}=1 \forall k \geq 1, a_{N+2 k+1}^{-}=1 \forall k \geq 0$, if $N$ is even,

$a_{N}^{-} \geq 2, a_{N}^{+}=a_{N}^{-}-1, a_{N+2 k}^{-}=1 \forall k \geq 1, a_{N+2 k+1}^{+}=1 \forall k \geq 0$, if $N$ is odd;

(iii) The pseudocenter $\bar{p} / \bar{q}$ of $V$ satisfies

$\bar{p} / \bar{q}=\left[a_{0}, \ldots, a_{N-1}, a_{N}^{+}\right]=\left[a_{0}, \ldots, a_{N-1}, a_{N}^{-}, 1\right], B_{f}\left(\left[a_{0}, \ldots, a_{N-1}, a_{N}^{+}\right]\right) \leq$ $t$, if $N$ is even,

$\bar{p} / \bar{q}=\left[a_{0}, \ldots, a_{N-1}, a_{N}^{+}, 1\right]=\left[a_{0}, \ldots, a_{N-1}, a_{N}^{-}\right], B_{f}\left(\left[a_{0}, \ldots, a_{N-1}, a_{N}^{-}\right]\right) \leq$ $t$, if $N$ is odd.

(iv) If $p / q \in V$ is a convergent of either $\xi^{+}$or $\xi^{-}$and $p / q \neq \bar{p} / \bar{q}$ then

$$
p / q=\left[a_{0}, \ldots, a_{N-1}, a_{N}^{ \pm}, \ldots, a_{D}^{ \pm}, 1\right],
$$

(where $D$ is odd if $p / q$ is a convergent of $\xi^{+}$and even if it is convergent of $\left.\xi^{-}\right)$and $B_{f}\left(\left[a_{0}, \ldots, a_{N-1}, a_{N}^{ \pm}, \ldots, a_{D}^{ \pm}, 1\right]\right) \leq t$ but $B_{f}\left(\left[a_{0}, \ldots, a_{N-1}\right.\right.$, $\left.\left.a_{N}^{ \pm}, \ldots, a_{D}^{ \pm}+1\right]\right)>t$.

(v) If $p / q \in] \bar{p} / \bar{q}, \xi^{+}\left[\right.$and $p / q$ is not a convergent for $\xi^{+}$then exists $p^{\prime} / q^{\prime}$ convergent of $\xi^{+}$such that $p / q<p^{\prime} / q^{\prime}<\xi^{+}$and $q^{\prime}<q$. Moreover, the value of the finite Brjuno function exceeds $t$ on both the continued fraction expansions of $p / q$. A similar statement holds in case $p / q \in] \xi^{-}, \bar{p} / \bar{q}[$, the only difference being that this time $p / q>p^{\prime} / q^{\prime}>\xi^{-}$.

(vi) If $p / q=\left[b_{0}, \ldots, b_{N}\right]$, with $b_{N}>1$, is a rational number such that $B_{f}\left(\left[b_{0}, \ldots, b_{N}\right]\right) \leq t$, then $p / q$ is the pseudocenter of the connected component of $A_{t}$ which contains it.

Proof. - In what follows we will only consider the case $N$ is even since the case $N$ odd is symmetric.

(i) $\xi^{ \pm} \notin A_{t}$ implies $\mathcal{B}\left(\xi^{ \pm}\right) \leq t$, on the other hand it cannot happen that $\mathcal{B}\left(\xi^{ \pm}\right)<t$ because otherwise, by Lemma 1 , one could find points in $A_{t}$ on which the value of $\mathcal{B}$ is strictly less than $t$ which is absurd.

(ii) Let $p_{N-1} / q_{N-1}=\left[a_{0}, \ldots, a_{N-1}\right]$ be the last rational which is a convergent of both $\xi^{ \pm}$and stays outside the interval $] \xi^{-}, \xi^{+}[$. Then:

$$
\begin{array}{r}
a_{N}^{+} \geq a_{N}^{-}+1, \\
\frac{p_{N}^{-}}{q_{N}^{-}}<\xi^{-}<\frac{p_{N+1}^{-}}{q_{N+1}^{-}} \leq \frac{p_{N}^{+}}{q_{N}^{+}}<\xi^{+}<\frac{p_{N-1}}{q_{N-1}} .
\end{array}
$$

Setting $\phi:=\left[a_{0}, \ldots, a_{N-1}, a_{N}^{+}-1+\phi_{0}\right]$, since $\xi^{+}>\phi$, and $\mathcal{B}(\phi)<\mathcal{B}\left(\xi^{+}\right) \leq t$, we get that $\xi^{-} \geq \phi$. Hence $a_{N}^{-} \geq a_{N}^{+}-1$, in fact by (8) equality holds.

If, by contradiction, $a_{N+2 k}^{+} \geq 2$ for some $k \geq 1$, setting

$$
\phi:=\left[a_{0}, \ldots, a_{N-1}, a_{N}^{+}, \ldots, a_{N+2 k}^{+}-1+\phi_{0}\right]
$$

BULletin DE LA Société MATHÉmatique DE FRANCE 
we would get $\xi^{-}<\phi<\xi^{+}$while $\mathcal{B}(\phi)<\mathcal{B}\left(\xi^{+}\right) \leq t$ which is impossible. An analogous argument shows that $a_{N+2 k+1}^{-}=1$ for all $k \geq 0$.

(iii) Let $\bar{p} / \bar{q}:=\left[a_{0}, \ldots, a_{N-1}, a_{N}^{+}\right]$. If $p / q \in V$ is a rational number and $p / q \neq$ $\bar{p} / \bar{q}$, then either $p / q \in I\left(a_{0}, \ldots, a_{N-1}, a_{N}^{+}\right)$or $p / q \in I\left(a_{0}, \ldots, a_{N-1}, a_{N}^{+}-1,1\right)$, and in both cases $q>\bar{q}$.

(iv) If $p / q$ is a convergent of $\xi^{+}$, and $p / q \neq \bar{p} / \bar{q}$, then $\left.p / q \in\right] \bar{p} / \bar{q}, \xi^{+}[$and

$$
p / q=\left[a_{0}, \ldots, a_{N-1}, a_{N}^{+}, \ldots, a_{N+2 d-1}^{+}, 1\right], \quad(d \geq 1) .
$$

Moreover, $B_{f}\left(\left[a_{0}, \ldots, a_{N-1}, a_{N}^{+}, \ldots, a_{N+2 d-1}^{+}, 1\right]\right) \leq t$. On the other hand, since

$$
\left.\left[a_{0}, \ldots, a_{N-1}, a_{N}^{+}, \ldots, a_{N+2 d-1}^{+}+1+\phi_{0}\right] \in\right] \bar{p} / \bar{q}, p / q\left[\subset A_{t},\right.
$$

it follows that

$$
\begin{aligned}
& B_{f}\left(\left[a_{0}, . ., a_{N-1}, a_{N}^{+}, \ldots, a_{N+2 d-1}^{+}+1\right]\right)= \\
& \quad \mathcal{B}\left(\left[a_{0}, . ., a_{N-1}^{+}, a_{N}^{+}, \ldots, a_{N+2 d-1}^{+}+1+\phi_{0}\right]\right)>t .
\end{aligned}
$$

If $p / q$ is a convergent for $\xi^{-}$the argument is symmetric.

(v) For $p / q \in] \bar{p} / \bar{q}, \xi^{+}\left[\right.$, and $p / q$ not a convergent for $\xi^{+}$, let

$$
\left[a_{0}, \ldots, a_{N-1}, a_{N}^{+}, \ldots, a_{N+2 d}^{+}\right]
$$

be the last convergent smaller than $p / q$, thus

$$
p / q=\left[a_{0}, \ldots, a_{N-1}, a_{N}^{+}, \ldots, a_{N+2 d}^{+}, b_{1}, \ldots, b_{H}\right]
$$

with $H \geq 1$. We claim that the rational

$$
\begin{aligned}
p^{\prime} / q^{\prime}=\left[a_{0}, . ., a_{N-1}, a_{N}^{+}, \ldots, a_{N+2 d}^{+}, a_{N+2 d+1}+1\right]= \\
\\
\quad\left[a_{0}, \ldots, a_{N-1}, a_{N}^{+}, \ldots, a_{N+2 d}^{+}, a_{N+2 d+1}, 1\right]
\end{aligned}
$$

is the convergent we are looking for. Indeed, by (i) $p^{\prime} / q^{\prime}$ is an even convergent and by assumption $p / q<p^{\prime} / q^{\prime}$ we deduce that $\left[b_{1}, \ldots, b_{H}\right] \geq a_{N+2 d+1}+1$, hence $b_{1} \geq a_{N+2 d+1}+1$.

Therefore

$$
\begin{aligned}
B_{f}\left(\left[a_{0}, . ., a_{N-1}, a_{N}^{+}, . .,\right.\right. & \left.\left.a_{N+2 d}^{+}, b_{1}, . ., b_{H}\right]\right) \geq \\
& B_{f}\left(\left[a_{0}, . ., a_{N-1}, a_{N}^{+}, \ldots, a_{N+2 d}^{+}, a_{N+2 d+1}+1\right]\right)>t,
\end{aligned}
$$

the last inequality being a consequence of (iii). If $p / q \in] \xi^{-}, \bar{p} / \bar{q}$ [ the proof can be carried over following the same argument.

(vi) This is a straightforward consequence of the previous statement.

REMARK. - $\mathcal{B}: \mathbb{R} / \mathbb{Z} \rightarrow[0,+\infty]$ is surjective. 
Remark. - From now on, if $r \in \mathbb{Q}$, by $B_{f}(r)$ we will mean the finite Brjuno function evaluated on the continued fraction expansion of $r$ which does not end with the figure 1 . By (vi) of Lemma 1 the set $\mathcal{Q}_{t}$ defined by

$$
\mathcal{Q}_{t}:=\left\{r \in \mathbb{Q}: B_{f}(r) \leq t\right\}
$$

is precisely the set of all pseudocenters of the connected components of $A_{t}$.

By this characterization it is clear that if $t \geq t_{0}$ then $\mathcal{Q}_{t} \supset \mathcal{Q}_{t_{0}}$. It is also interesting and important for the sequel to analyze the process of disintegration of the connected components of $A_{t}$; more precisely let $p / q \in \mathcal{Q}_{t_{0}}$ and $] \xi_{t}^{-}, \xi_{t}^{+}$[ be the connected component of $A_{t}$ of pseudocenter $p / q$ : the function $t \mapsto \xi_{t}^{+}-\xi_{t}^{-}$ is decreasing and has jumps exactly at those values of $t$ which are image under $\mathcal{B}$ of local minima in $] \xi_{t_{0}}^{-}, \xi_{t_{0}}^{+}[$.

Proposition 2.1. - If $t \geq 2 t_{0}$ then all convergents of $\xi_{t_{0}}^{ \pm}$belong to $\mathcal{Q}_{t}$.

Proof. - Indeed, if $p / q$ is such a convergent (which is not the pseudocenter of ]$\xi_{t_{0}}^{-}, \xi_{t_{0}}^{+}[)$, then

$$
\begin{aligned}
& p / q=\left[a_{0}, \ldots, a_{N-1}, a_{N}^{ \pm}, \ldots, a_{D}^{ \pm}, 1\right], \\
& \text { where } D \geq 1 \text { is }\left\{\begin{array}{l}
\text { odd for } \xi^{+}, \\
\text {even for } \xi^{-} .
\end{array}\right.
\end{aligned}
$$

Note that, since $\max \left(a_{N}^{+}, a_{N}^{-}\right) \geq 2$, we have that

$$
t_{0} \geq \max \left(\mathcal{B}\left(\xi^{+}\right), \mathcal{B}\left(\xi^{-}\right)\right) \geq \frac{\log 2}{q_{N-1}} .
$$

So, assuming for the sake of simplicity that $p / q$ is a convergent of $\xi^{+}$and dropping the superscript + , we can readily check that $B_{f}\left(\left[a_{0}, \ldots, a_{N}, \ldots, a_{D}+\right.\right.$ 1]) $\leq t$, which implies that $p / q \in \mathcal{Q}_{t}$ :

$$
\begin{aligned}
B_{f}\left(\left[a_{0}, \ldots, a_{D}+1\right]\right) & =\sum_{k=0}^{D-2} \frac{\log a_{k+1}}{q_{k}}+\frac{\log \left(a_{D}+1\right)}{q_{D-1}} \\
& =\sum_{k=0}^{D-1} \frac{\log a_{k+1}}{q_{k}}+\frac{\log \left(1+a_{D}^{-1}\right)}{q_{D-1}} \\
& \leq t_{0}+\frac{\log 2}{q_{N-1}} \leq 2 t_{0} \leq t .
\end{aligned}
$$

Let $M>0$ be fixed. Let $V^{*}$ be a connected component of $A_{M}$ and let $V$ be a connected component of $A_{3 M}$ contained in $V^{*}$. By the previous remarks we have

$$
\begin{array}{r}
\left.V^{*}=\right] \alpha^{-}, \alpha^{+}\left[, \text {with } \mathcal{B}\left(\alpha^{ \pm}\right)=M\right. \\
V=] \zeta^{-}, \zeta^{+}\left[, \text {with } \mathcal{B}\left(\zeta^{ \pm}\right)=3 M\right.
\end{array}
$$


We now shall establish a lower bound for the quantities $\left|\alpha^{+}-\zeta^{+}\right|$and $\left|\alpha^{-}-\zeta^{-}\right|$. As usual we will carry over the caculations only for the bound on $\left|\alpha^{+}-\zeta^{+}\right|$, the other case being analogous. Set $\alpha^{+}:=\left[a_{0}, \ldots, a_{N}, \ldots\right], \zeta^{+}:=$ $\left[c_{0}, \ldots, c_{N-1}, c_{N}, c_{N+1}, 1, c_{N+3}, 1, \ldots\right]$ and let $p / q=\left[c_{0}, \ldots, c_{N-1}, c_{N}\right]$ be the pseudocenter of the interval $] \zeta^{-}, \zeta^{+}[$; we will distinguish the following cases:

Case $A: p / q$ is a convergent of $\alpha^{+}$as well.

In this case $a_{k}=c_{k}$ for all $1 \leq k \leq N$. Setting $\phi:=\left[c_{0}, . ., c_{N-1}, c_{N}, 2 c_{N+1}, 1,1,1\right.$, it is immediate to check that $p / q<\phi<\zeta^{+}$and hence $\mathcal{B}(\phi)>3 M$. Since

$$
\mathcal{B}(\phi)=\sum_{k=0}^{N-1} \frac{\log c_{k+1}}{q_{k}}+\frac{\log 2 c_{N+1}}{q_{N}} \leq \mathcal{B}\left(\alpha^{+}\right)+\frac{\log 2 c_{N+1}}{q_{N}} \leq M+\frac{\log 2 c_{N+1}}{q_{N}}
$$

it follows that $2 c_{N+1}>e^{2 M q_{N}}$ and so

$$
\left|\zeta^{+}-p / q\right| \leq \frac{1}{c_{N+1} q^{2}} \leq \frac{2 e^{-2 M q}}{q^{2}}
$$

Since $\mathcal{B}\left(\alpha^{+}\right) \leq M$, we get $q_{N}^{-1} \log \left(a_{N+1}\right) \leq M$ and hence $a_{N+1} \leq e^{M q_{N}}$. So

$$
\left|\alpha^{+}-p / q\right| \geq \frac{1}{2 a_{N+1} q^{2}} \geq \frac{e^{-M q}}{2 q^{2}}
$$

Using (10) and (11) we gain

$$
\alpha^{+}-\zeta^{+} \geq \alpha^{+}-p / q-\left(\zeta^{+}-p / q\right) \geq \frac{e^{-M q}}{2 q^{2}}\left(1-4 e^{-M q}\right),
$$

and

$$
\alpha^{+}-\zeta^{+} \geq \frac{e^{-M q}}{4 q^{2}} \quad \text { holds as soon as } \quad q \geq \frac{\log 8}{M}
$$

Case $B: p / q$ is not a convergent of $\alpha^{+}$.

If $p / q$ is not a convergent of $\alpha^{+}$then there is some convergent $r / s$ of $\alpha^{+}$ such that

$$
p / q<r / s<\alpha^{+} \quad \text { and } \quad s<q,
$$

hence

$$
\alpha^{+}-\zeta^{+} \geq \alpha^{+}-r / s \geq \frac{e^{-M s}}{2 s^{2}} \geq \frac{e^{-M q}}{2 q^{2}}
$$

The same estimates hold also for $\left|\alpha^{-}-\zeta^{-}\right|$so, putting together the cases $\mathrm{A}$ and $\mathrm{B}$, we gain the following lemma 
LEMMA 5. - There exists a positive constant $\nu_{0}$ such that if $V$ is a connected component of $A_{3 M}, p / q$ is the pseudocenter of $V, \zeta \in \partial V$ and $\mathcal{B}(\alpha) \leq M$ one has

$$
|\alpha-\zeta| \geq \nu_{0} \frac{e^{-M q}}{q^{2}} .
$$

Proof. - The proof is straightforward: it is sufficient to note that the results above imply that (13) holds with $\nu_{0}=1 / 4$ for all but finitely many connected components of $A_{3 M}$. Therefore choosing $\nu_{0}$ sufficiently small we establish that (13) holds with no exceptions.

\section{3. $\mathcal{C}^{1}$-holomorphic and monogenic regularity of the conjugation}

The main result we shall prove in this section is that $H \in \mathcal{C}_{\text {hol }}^{1}\left(C_{M} ; \mathcal{H}^{\infty}\left(\mathbb{D}_{\rho}\right)\right)$, where $C_{M}$ is a set obtained removing from the complex plane $\mathbb{C}$ the union of tiny neighbourhoods of the roots of unity while $\rho>0$ is suitably chosen. Let us begin describing the "domain of regularity" $C_{M}$.

3.1. Domain of regularity. - Let $\kappa \in] 0,1[$ be fixed; if $V=] \xi^{+}, \xi^{-}[$is an open interval in $\mathbb{R} / \mathbb{Z}$ we shall call $\kappa$-diamond ${ }^{(6)}$ on $V$ the set

$$
\Delta:=\left\{z \in \mathbb{C} / \mathbb{Z}: \xi^{-}<\Re(z)<\xi^{+} \text {and }|\Im(z)|<\kappa \min \left(\Re z-\xi^{-}, \xi^{+}-\Re z\right)\right\} .
$$

Let $M>0$ be fixed. Let $\mathcal{Q}_{M}$ be the set of pseudocenters of connected components of the open superlevel $A_{M}$ of the Brjuno function and let $\Delta(M, r)$ be the diamond on the connected component of $A_{M}$ containing $r$; it is then easy to check that

$$
\Omega_{M}:=\bigcup_{r \in \mathcal{Q}_{M}} \Delta(M, r)
$$

is an open neighbourhood of $\mathbb{Q} / \mathbb{Z}$ in $\mathbb{C} / \mathbb{Z}$. Hence $K_{M}:=(\mathbb{C} / \mathbb{Z}) \backslash \Omega_{M}$ is a closed set which does not contain any rational number. Moreover it is straightforward to check that any of diophantine sets $D C(\gamma, \tau):=\{\alpha \in \mathbb{R} / \mathbb{Z}|| \alpha-p / q \mid \geq$ $\left.\gamma / q^{-\tau} \forall p / q \in \mathbb{Q}\right\}$ is contained in some $K_{M}$ for $M$ sufficently large. Since for any fixed $\tau>2$ meas $_{1}(\mathbb{R} / \mathbb{Z} \backslash D C(\gamma, \tau)) \rightarrow 0$ as $\gamma \rightarrow 0$ it follows that $\operatorname{meas}_{2}\left(\mathbb{C} / \mathbb{Z} \backslash K_{M}\right) \rightarrow 0$ as $M \rightarrow+\infty$. It could also be proved (see [11]) that meas $\left(K_{M}\right)>0$ for all $M>0$ and in fact each point $x \in K_{M}$ either is isolated (in the exceptional case when $x$ is a local minimunm for the Brjuno function) or $x$ is a point of density for $K_{M}$.

Let $\exp ^{\#}: \mathbb{C} / \mathbb{Z} \rightarrow \mathbb{C}^{*}$ be defined as $\exp ^{\#}(\zeta):=\exp (2 \pi i \zeta)$. For $d>0$ we define $S_{d}:=\{\zeta \in \mathbb{C} / \mathbb{Z}:|\Im(\zeta)| \leq d\}$ the strip of height $2 d$ around the real axis and the annulus $\exp ^{\#}\left(S_{d}\right):=\mathbb{A}_{d}$. We point out that the restriction

(6) Or, simply, diamond, since we shall not play with different values of $\kappa$. 
$\exp ^{\#}: S_{d} \rightarrow \mathbb{A}_{d}$ is a covering map and locally a biholomorphism, hence by compactness there exists $\eta>1$ (depending on $d$ ) such that

$$
\eta^{-1}\left|\zeta-\zeta^{\prime}\right| \leq\left|\exp ^{\#}(\zeta)-\exp ^{\#}\left(\zeta^{\prime}\right)\right| \leq \eta\left|\zeta-\zeta^{\prime}\right| \quad \forall \zeta, \zeta^{\prime} \in S_{d}
$$

The set $C_{M}:=\exp ^{\#}\left(K_{M}\right) \cup\{0, \infty\}$ is the domain on which the conjugation $H$ will be "regular".

Proposition 3.1. - There exists a universal constant $b_{1}$ such that for all values of the multiplier $\lambda \in C_{M}$ the power series expansion of the conjugation

$$
H_{\lambda}(z)=\sum_{k=0}^{+\infty} h_{k}(\lambda) z^{k}
$$

has radius of convergence at least $e^{-\left(M+b_{1}\right)}>0$. Moreover $\max _{\lambda \in C_{M}}\left|h_{k}(\lambda)\right| \leq$ $e^{\left(M+b_{1}\right) k}$.

Proof. - We point out that for any fixed $d>0$, if $\lambda \notin \mathbb{A}_{d}$ then no small divisor occours in the recurrence (3) and the thesis is a straightforward consequence of the classical majorant series method. Nevertheless the estimates we get depend on $d$ and deteriorate as $\lambda$ approaches the unit circle. Therefore we only have to check the statement when $\lambda$ is in some annulus around the unit circle. For this reason in the following we fix $d>0$ and we consider only those values of the paramenter $\lambda$ which can be written as $\lambda=e^{2 \pi i \xi}, \xi \in K_{M},|\Im \xi| \leq d$. We can associate to any such $\xi$ a point $\xi_{0} \in K_{M} \cap \mathbb{R}$ in the following way: $\xi_{0}:=\Re(\xi)$ if $\Re(\xi) \notin A_{M}$ while, if $\Re(\xi)$ belongs to the connected component ] $\xi^{-}, \xi^{+}\left[\right.$of $A_{M}$, we shall choose $\xi_{0}$ to be the nearest point to $\Re(\xi)$ among the two values $\xi^{+}$or $\xi^{-}$; we define also $\lambda_{0}=e^{2 \pi i \xi_{0}}$.

In this way we can easily check that

$$
\left|\lambda^{k}-1\right| \geq \delta\left|\lambda_{0}^{k}-1\right|, \quad \forall \lambda \in C_{M},
$$

where $\delta:=\min \left(e^{-d} \eta^{-2}\left(1+\kappa^{-2}\right)^{-\frac{1}{2}}, \frac{1-e^{-d}}{2}\right)$. By the recurrence relation (3) we get that

$$
\left|h_{k}(\lambda)\right| \leq \delta^{-k}\left|h_{k}\left(\lambda_{0}\right)\right|
$$

By the Brjuno estimate (4) (see also [4] for its proof) we deduce that for all $\lambda_{0} \in C_{M} \cap \mathbb{S}^{1}$ the radius of convergence $\rho\left(\lambda_{0}\right)$ of the series $H_{\lambda_{0}}(z)=$ $\sum_{k=0}^{+\infty} h_{k}\left(\lambda_{0}\right) z^{k}$ satisfies $\rho\left(\lambda_{0}\right) \geq e^{-\left(M+b_{0}\right)}>0$. So, by (16), we get that for all $\lambda \in C_{M} \cap \mathbb{A}_{d}$ the radius of convergence of series $H_{\lambda}(z)=\sum_{k=0}^{+\infty} h_{k}(\lambda) z^{k}$ is greater than $\delta^{-1} e^{-\left(M+b_{0}\right)}$.

From now on we set $d:=\kappa / 2$, so that $\Delta(3 M, p / q) \subset S_{d}$ for all $p / q \in \mathbb{Q}$ and $\eta$ will be the constant appearing in (14) relative to $S_{d}$.

TOME $136-2008-\mathrm{N}^{\mathrm{O}} 4$ 
Lemma 6. - Let $p / q \in \mathbb{Q}, \Delta(3 M, p / q)$ be the diamond on the connected component of the superlevel $A_{3 M}$ containing $p / q$ and let $D(3 M, p / q):=$ $\exp ^{\#}(\Delta(3 M, p / q))$. Then, if $\lambda \in C_{M}$

$$
d(\lambda, \partial D(3 M, p / q)) \geq \nu_{1} \frac{e^{-M q}}{q^{2}}
$$

where $\nu_{1}=\frac{\nu_{0}}{\eta} \frac{\kappa}{\sqrt{1+\kappa^{2}}}$

Proof. - Let $\lambda=e^{2 \pi i \alpha}, \alpha \in K_{M}$, we immediately get

$$
d(\lambda, \partial D(3 M, p / q)) \geq \eta^{-1} d(\alpha, \partial \Delta(3 M, p / q)) \geq \nu_{1} \frac{e^{-M q}}{q^{2}},
$$

where the last inequality follows from Lemma 5 together with an elementary geometrical argument.

3.2. $\mathcal{C}_{h o l}^{1}$-regularity. - With a slight abuse of notation let us set $H_{k}:=$ $\max _{\lambda \in C_{3 M}}\left|h_{k}(\lambda)\right|$; we know from Proposition 3.1 that the series $\sum H_{k} z^{k}$ has a positive radius of convergence bounded from below by $\rho_{0}:=e^{-\left(3 M+b_{1}\right)}$. The main result in this section is the following:

ThEOREM 1. - Let $\rho \in] 0, e^{-2 M} \rho_{0}\left[\right.$. Then the map $h: \lambda \mapsto H_{\lambda}$ belongs to the space of functions $\mathcal{C}_{\text {hol }}^{1}\left(C_{M} ; \mathcal{H}^{\infty}\left(\mathbb{D}_{\rho}\right)\right)$.

We already know that, by virtue of Proposition 3.1,

$$
H_{\lambda}(z)=\sum_{k=1}^{+\infty} h_{k}(\lambda) z^{k}
$$

has positive radius of convergence for $\lambda \in C_{M}$, moreover each of the coefficients $h_{k}$, defined by the recurrence relation (3), is a rational function in the variable $\lambda$ and is holomorphic away from the roots of unity of order strictly less than $k$.

In order to prove the theorem we shall show that the series (17) is normally convergent in $\mathcal{C}_{h o l}^{1}\left(C_{M} ; \mathcal{H}^{\infty}\left(\mathbb{D}_{\rho}\right)\right)$; and this will be a straightforward consequence of point (iii) of the next lemma:

LEMMA 7. - There exists a positive constant $L>1$ such that

(i) $\left|h_{k}^{\prime}(\lambda)\right| \leq L\left(1+k^{4}\right) H_{k} e^{2 M k} \quad \forall \lambda \in C_{M}$

(ii) $\left|\frac{h_{k}\left(\lambda_{1}\right)-h_{k}\left(\lambda_{0}\right)}{\lambda_{1}-\lambda_{0}}-h^{\prime}\left(\lambda_{0}\right)\right| \leq 2 L\left(1+k^{4}\right) H_{k} e^{2 M k} \quad \forall \lambda_{1}, \quad \lambda_{0} \in C_{M}$

(iii) $\left\|h_{k}(\lambda) z^{k}\right\|_{\mathcal{C}_{h o l}^{1}\left(C_{M} ; \mathcal{H}^{\infty}\left(\mathbb{D}_{\rho}\right)\right)} \leq 4 L\left(1+k^{4}\right) H_{k}\left(e^{2 M} \rho\right)^{k}$. 
Proof. - From now on $r$ will always denote a rational number and $\operatorname{ord}(r):=$ $\min \left\{n \in \mathbb{N}^{*}: n r \in \mathbb{Z}\right\} ;$ moreover we use the following notation:

$$
\begin{aligned}
& \mathbb{Q}_{k}=\{r \in \mathbb{Q}: \operatorname{ord}(r)<k\} ; \quad D_{r}:=\exp ^{\#}(\Delta(3 M, r)) \\
& \mathcal{Q}_{t, k}:=\left\{r \in \mathbb{Q}: B_{f}(r)<t\right\} \cap \mathbb{Q}_{k} ;
\end{aligned}
$$

Since $h_{k}$ is a rational function with poles located on the roots of unity of order less than $k$

$$
\mathcal{R}_{k}:=\exp ^{\#}\left(\mathbb{Q}_{k}\right) \subset \bigcup_{r \in \mathcal{Q}_{3 M, k}} D_{r},
$$

for any $R>|\lambda|$ we get, by Cauchy formula,

$$
h_{k}(\lambda)=\sum_{r \in \mathcal{Q}_{3 M, k}} \frac{1}{2 \pi i} \int_{\partial D_{r}} \frac{h_{k}(\zeta)}{\zeta-\lambda} d \zeta+\frac{1}{2 \pi i} \int_{\partial \mathbb{D}_{R}} \frac{h_{k}(\zeta)}{\zeta-\lambda} d \zeta .
$$

In fact, letting $R \rightarrow+\infty$, we realize that the term $\int_{\partial \mathbb{D}_{R}} \frac{h_{k}(\zeta)}{\zeta-\lambda} d \zeta$ must vanish; hence in the following we will always neglect this term. We can wrtite the integral representation both for the derivative of $h_{k}$ and for the Taylor remainder $R_{2}\left(h_{k}, \lambda_{0}, \lambda_{1}\right):=h_{k}\left(\lambda_{1}\right)-h_{k}\left(\lambda_{0}\right)-h_{k}^{\prime}\left(\lambda_{0}\right)\left(\lambda_{1}-\lambda_{0}\right):$

$$
\begin{aligned}
& \mid\left(\lambda_{1}-\lambda_{0}\right)^{-1} R_{2}\left(h_{k}, \lambda_{0}, \lambda_{1}\right) \mid= \\
& \sum_{s \in \mathcal{Q}_{3 M, k}} \frac{1}{2 \pi i} \int_{\partial D_{s}}\left[\frac{1}{\left(\zeta-\lambda_{1}\right)\left(\zeta-\lambda_{0}\right)}-\frac{1}{\left(\zeta-\lambda_{0}\right)^{2}}\right] h_{k}(\zeta) d \zeta .
\end{aligned}
$$

So we get the estimates

$$
\begin{gathered}
\left|h_{k}^{\prime}(\lambda)\right| \leq \frac{H_{k}}{2 \pi} \sum_{s \in \mathcal{Q}_{3 M, k}} d\left(\lambda, \partial D_{s}\right)^{-2} \int_{D_{s}}|d \zeta|, \\
\left|\left(\lambda_{1}-\lambda_{0}\right)^{-1} R_{2}\left(h_{k}, \lambda_{0}, \lambda_{1}\right)\right| \leq \frac{H_{k}}{2 \pi} \sum_{s \in \mathcal{Q}_{3 M, k}} 2 d\left(\lambda, \partial D_{s}\right)^{-2} \int_{D_{s}}|d \zeta| .
\end{gathered}
$$

Lemma 5 gives an upper bound on the term $d\left(\lambda, \partial D_{s}\right)^{-2}$ while

$$
\int_{D_{s}}|d \zeta| \leq \eta \int_{\Delta_{s}}|d z| \leq 2 \eta\left(1+\kappa^{-2}\right)^{1 / 2}\left|V_{s}\right| \quad \text { for } \quad s \in \mathcal{Q}_{3 M, k} .
$$

TOME $136-2008-\mathrm{N}^{\mathrm{O}} 4$ 
Since $\sum_{s \in \mathcal{Q}_{3 M, k}}\left|V_{s}\right|<1$ we finally get

$$
\begin{aligned}
\left|h_{k}^{\prime}(\lambda)\right| & \leq \frac{H_{k}}{2 \pi}\left[2 \eta\left(1+\kappa^{-2}\right)^{1 / 2} \nu_{1}^{-2} k^{4} e^{M k}\right], \\
\left|\left(\lambda_{1}-\lambda_{0}\right)^{-1} R_{2}\left(h_{k}, \lambda_{0}, \lambda_{1}\right)\right| & \leq \frac{H_{k}}{\pi}\left[2 \eta\left(1+\kappa^{-2}\right)^{1 / 2} \nu_{1}^{-2} k^{4} e^{M k}\right] .
\end{aligned}
$$

The thesis follows choosing $L:=\max \left\{1, \frac{\eta}{\pi}\left(1+\kappa^{-2}\right)^{1 / 2} \nu_{1}^{-2}\right\}$.

3.3. Monogenic regularity.- We refer the reader to Appendix B for a more detailed treatment of monogenic functions. Let us choose an increasing sequence of positive values $M_{j}$ such that $\lim M_{j}=+\infty$ and set $C_{j}:=C_{M_{j}}$.

Consider the Banach space $B_{\ell}=\cap_{j=0}^{\ell} \mathcal{C}_{h o l}^{1}\left(C_{j}, \mathcal{H}^{\infty}\left(\mathbb{D}_{r_{j}}\right)\right)$, where $r_{j}=$ $e^{-\left(3 M_{j}+b_{1}\right)}$ with the norm $\|f\|_{B_{\ell}}=\max _{0 \leq j \leq l}\|f\|_{\mathcal{C}_{h o l}^{1}\left(C_{j}, \mathcal{H}^{\infty}\left(\mathbb{D}_{r_{j}}\right)\right)}$. Clearly the injections $i_{\ell}: B_{\ell} \hookrightarrow B_{\ell-1}$ are bounded linear operators between Banach spaces with norms $\left\|i_{\ell}\right\| \leq 1$. The projective limit of the system of Banach spaces

$$
\mathcal{M}\left(\left(C_{\ell}\right), \mathbb{C}\{z\}\right)=\lim _{\longleftarrow} B_{\ell}
$$

is a space of monogenic functions with values in the holomorphic germs $\mathbb{C}\{z\}$. This is a Fréchet space with the family of seminorms $\left(\|\cdot\|_{B_{\ell}}\right)_{\ell \in \mathbb{N}}$.

Thus Theorem 1 has the following corollary

Corollary 8. - Let $\left(C_{\ell}\right)$ be as above. Then the linearization $H$ belongs to the space $\mathcal{M}\left(\left(C_{\ell}\right), \mathbb{C}\{z\}\right)$ of $\mathbb{C}\{z\}$-valued monogenic functions.

\section{Higher regularity}

Using the Cauchy formula (19) we get an integral representation for the $m$-th derivative of $h_{k}$ as well:

$$
h_{k}^{(m)}(\lambda)=\sum_{s \in \mathcal{Q}_{3 M, k}} \frac{m !}{2 \pi i} \int_{\partial D_{s}} \frac{h_{k}(\zeta)}{(\zeta-\lambda)^{m+1}} d \zeta .
$$

It is quite easy to see that, if we just followed the same lines of the previous section, in order to gain $\mathcal{C}_{h o l}^{m}$ regularity we would have to shrink the radius $\rho$ of the disk $\mathbb{D}_{\rho}$ and trying to prove that $h$ is $C^{\infty}$-holomorphic would lead to a disk of convergence of radius zero.

To avoid this problem we use an idea of [13]: we will prove that $h \in$ $C_{\text {hol }}^{\infty}\left(C^{*}, \mathcal{H}^{\infty}\left(\mathbb{D}_{\rho}\right)\right)$ for some $\rho>0$, where this time $C^{*}$ will be somewhat smaller than the set $C_{M}$ considered in the previous section. 
4.1. Construction of the domain $C^{*}$. - Let $\left(M_{n}\right)_{n \in \mathbb{N}}$ be a decreasing sequence such that $M_{n} \rightarrow 0$ as $n \rightarrow \infty$ and define the set

$$
K_{\left(M_{n}\right)}^{*}:=\left\{x \in \mathbb{R} / \mathbb{Z}: \sum_{k=n}^{+\infty} \frac{\log a_{k+1}}{q_{k}} \leq M_{n} \forall n \in \mathbb{N}\right\} .
$$

Of course $K_{\left(M_{n}\right)}^{*} \subset K_{M}$ for $M=M_{0}$ and it is not difficult to check that $K_{\left(M_{n}\right)}$ is compact in $\mathbb{R} / \mathbb{Z}$. It is clear that $K_{\left(M_{n}\right)} \neq \varnothing$ and it is possible to choose $M_{n} \rightarrow 0$ such that meas $\left(K_{\left(M_{n}\right)}^{*}\right)>0$ : in fact for all $\tau, \gamma$ there exists $M_{n} \rightarrow 0$ such that $K_{\left(M_{n}\right)}^{*} \supset D C(\gamma, \tau)$ (see also Section 5). As before, we define $A^{*}:=(\mathbb{R} / \mathbb{Z}) \backslash K_{\left(M_{n}\right)}^{*}$ and we denote with $\mathcal{Q}^{*}$ the set of all pseudocenters of the connected components of $A^{*}$. We now have a list of technical lemmata that will be useful later on.

LEMmA 9. - There exists a function $Q: \mathbb{N} \rightarrow \mathbb{R}$ such that if $\alpha:=$ $\left[a_{0}, \ldots, a_{n}, \ldots\right] \in K_{\left(M_{n}\right)}^{*}$, and $p_{n} / q_{n}=\left[a_{0}, \ldots, a_{n}\right]$ is the $n$-th convergent then $F_{n} \leq q_{n} \leq Q_{n}$ (where $F_{n}$ are, as usual, the Fibonacci numbers).

Proof. - The sequence $Q_{n}$ defined by the recurrence

$$
\left\{\begin{array}{l}
Q_{0}=1, \\
Q_{n+1}=e^{M_{n} Q_{n}} Q_{n}+Q_{n-1},
\end{array}\right.
$$

does the job.

Next lemma is almost a clone of Lemma 4.

Lemma 10. - Let $\left.V^{*}=\right] \alpha^{-}, \alpha^{+}\left[\right.$be a connected component of $A^{*}$ and let

$$
\alpha^{ \pm}:=\left[a_{0}, . ., a_{N-1}, a_{N}^{ \pm}, a_{N+1}^{ \pm}, \ldots\right], \quad N \geq 1, \quad a_{N}^{+} \neq a_{N}^{-} .
$$

Then

(i) $a_{N}^{+} \geq 2, a_{N}^{-}=a_{N}^{+}-1, a_{N+2 k}^{+}=1 \forall k \geq 1, a_{N+2 k+1}^{-}=1 \forall k \geq 0$, if $N$ is even,

$a_{N}^{-} \geq 2, a_{N}^{+}=a_{N}^{-}-1, a_{N+2 k}^{-}=1 \forall k \geq 1, a_{N+2 k+1}^{+}=1 \forall k \geq 0$, if $N$ is odd;

(ii) There is a unique rational number $\bar{p} / \bar{q}$ which is a convergent of both $\alpha^{ \pm}$; $\bar{p} / \bar{q}$ is the rational number with lowest denominator in $V^{*}$ (it is then the pseudocenter of $V^{*}$ ) and

$\bar{p} / \bar{q}=\left[a_{0}, \ldots, a_{N-1}, a_{N}^{+}\right]=\left[a_{0}, \ldots, a_{N-1}, a_{N}^{-}, 1\right], B_{f}\left(\left[a_{0}, \ldots, a_{N-1}, a_{N}^{+}\right]\right) \leq$ $M_{0}$, if $N$ is even,

$\bar{p} / \bar{q}=\left[a_{0}, \ldots, a_{N-1}, a_{N}^{+}, 1\right]=\left[a_{0}, \ldots, a_{N-1}, a_{N}^{-}\right], B_{f}\left(\left[a_{0}, \ldots, a_{N-1}, a_{N}^{-}\right]\right) \leq$ $M_{0}$, if $N$ is odd; 
(iii) If $p / q \in V^{*}$ is a convergent of either $\alpha^{+}$or $\alpha^{-}$and $p / q \neq \bar{p} / \bar{q}$ then

$$
p / q=\left[a_{0}, \ldots, a_{N-1}, a_{N}^{ \pm}, \ldots, a_{D}^{ \pm}, 1\right]
$$

(where $D$ is odd if $p / q$ is a convergent of $\alpha^{+}$and even if it is convergent of $\left.\alpha^{-}\right)$.

(iv) If $p / q \in] \bar{p} / \bar{q}, \alpha^{+}\left[\right.$and $p / q$ is not a convergent for $\alpha^{+}$then exists $p^{\prime} / q^{\prime}$ convergent of $\alpha^{+}$such that $p / q<p^{\prime} / q^{\prime}<\alpha^{+}$and $q^{\prime}<q$. A similar statement holds in case $p / q \in] \alpha^{-}, \bar{p} / \bar{q}[$, the only difference being that this time $p / q>p^{\prime} / q^{\prime}>\alpha^{-}$.

(v) Any convergent of $\alpha^{ \pm}$is a pseudocenter of some connected component of $A_{3 M_{0}}$ (hence belongs to $\mathcal{Q}_{3 M_{0}}$ ).

Proof. - We just sketch some details, since the whole proof of (i)-(iv) is just a repetition the arguments of Lemma 4 while (v) follows from the same argument as in Proposition 2.1. Let $V^{*}=\left(\alpha^{-}, \alpha^{+}\right)$be a connected component of $A^{*}$ and let $\bar{r}$ be the pseudocenter of $V^{*}$. Write $\bar{r}=\left[a_{0}, \ldots, a_{N}\right]$ with $a_{N} \geq 2$ and assume, just to fix ideas, that $N$ is even. Letting $r^{-}:=\left[a_{0}, \ldots, a_{N-1}\right]$ and $r^{+}:=\left[a_{0}, \ldots, a_{N}-1\right]$, it is readily checked that $\operatorname{ord}\left(r^{ \pm}\right)<\operatorname{ord}(\bar{r})$ and $r^{-}<\bar{r}<r^{+}$, hence, by the minimality of the order of $\bar{r}, V^{*} \subset\left(r^{-}, r^{+}\right)$. On the other hand, for any fixed $n \in \mathbb{N}$, the expression $\sum_{k=n}^{+\infty} \frac{\log a_{k+1}}{q_{k}}$ attains its minimum value on the interval $\left(\bar{r}, r^{+}\right)$at the point $\phi^{+}:=\left[a_{0}, \ldots, a_{N}+\phi_{0}\right]$ while the minimum value on $\left(r^{-}, \bar{r}\right)$ is attained at $\phi^{-}:=\left[a_{0}, \ldots, a_{N}-1+\phi_{0}\right]$. This implies that $\left(\alpha^{-}, \alpha^{+}\right) \subset\left(\phi^{-}, \phi^{+}\right)$and $\bar{r}=\left[a_{0}, \ldots, a_{N}\right]$ is a common convergent of both $\alpha^{ \pm}$.

Let $k \in \mathbb{N}$ be fixed, $r \in \mathcal{Q}^{*} \cap \mathbb{Q}_{k}$ (recall the definitions (18)) and let $V_{r}^{*}=$ ]$\alpha^{-}, \alpha^{+}$[ be the connected component of $A^{*}$ with pseudocenter $r$. Let us define

$$
s^{+}(k, r):=\max \left\{s \in \mathbb{Q}_{k} \cap\right] \alpha^{-}, \alpha^{+}[\}, \quad s^{-}(k, r):=\min \left\{s \in \mathbb{Q}_{k} \cap\right] \alpha^{-}, \alpha^{+}[\} .
$$

By virtue of (iv) of the previous lemma $s^{+}$is a convergent of $\alpha^{+}$, while $s^{-}$is a convergent of $\alpha^{-}$therefore, by (v) of the previous lemma, both $s^{ \pm}$belong to $\mathcal{Q}_{3 M_{0}, k}$. Let

$$
s(k, r):= \begin{cases}s^{+}(k, r) & \text { if } d\left(\alpha^{+}, \partial V_{s^{+}(k, r)}\right)<d\left(\alpha^{-}, \partial V_{s^{-}(k, r)}\right) \\ s^{-}(k, r) & \text { if } d\left(\alpha^{+}, \partial V_{s^{+}(k, r)}\right) \geq d\left(\alpha^{-}, \partial V_{s^{-}(k, r)}\right)\end{cases}
$$

and $q(k, r):=\operatorname{ord}(s(k, r))<k$.

Note that if $r \in \mathcal{Q}^{*} \cap \mathbb{Q}_{k}$ then ord $(r) \leq q(k, r)<k$

Thus, if $s \in \mathcal{Q}_{3 M_{0}, k} \cap V_{r}^{*}$ and $V_{s}$ is the connected component of $A_{3 M_{0}}$ of pseudocenter $s=p / q$, we have that

$$
d\left(\alpha, \partial V_{s}\right) \geq d\left(\alpha, \partial V_{s(k, r)}\right) .
$$


The first part of next lemma is just Lemma 5 , the second contains the extra information we shall need to prove that $h$ is $\mathcal{C}_{h o l}^{\infty}$.

LEMMA 11. - 1. Let $s \in \mathcal{Q}_{3 M_{0}}$ and $V_{s}$ be the connected component of pseudocenter $s=p / q$, then

$$
d\left(\alpha, \partial V_{s}\right) \geq \nu_{0} \frac{e^{-M_{0} q}}{q^{2}}, \quad \forall \alpha \in K_{\left(M_{n}\right)}^{*}
$$

(where $\nu_{0}$ is a constant independent of $s$ ).

2. Let $\left.n, k \in \mathbb{N}, V_{r}^{*}=\right] \alpha^{-}, \alpha^{+}\left[\right.$be the connected component of $A^{*}$ with pseudocenter $r$ and assume that $q(k, r) \geq \max \left\{Q_{n}, \frac{\log 8}{M_{0}}\right\}$. If $s \in \mathcal{Q}_{3 M_{0}, k} \cap V_{r}^{*}$ then

$$
d\left(\alpha, \partial V_{s}\right) \geq \frac{e^{-M_{n} k}}{4 k^{2}} \quad \forall \alpha \in K^{*}\left(M_{n}\right) .
$$

Proof. - We just have to prove the second statement; for sake of simplicity let us assume that $s(k, r)=s^{+}(k, r)$, the other case being analogous. If $s \in$ $\mathcal{Q}_{3 M_{0}, k} \cap V_{r}^{*}$ then

$$
d\left(\alpha, \partial V_{s}\right) \geq d\left(\alpha, \partial V_{s(k, r)}\right) \geq d\left(\alpha^{+}, \partial V_{s^{+}(k, r)}\right) .
$$

Setting $\left.V_{s^{+}(k, r)}:=\right] \zeta^{-}, \zeta^{+}\left[, s^{+}(k, r):=p / q\right.$ (so that $\left.q=q(k, r) \geq Q_{n}\right)$ we can repeat the argument of the end of subsection 3.1:

$$
\begin{aligned}
\left|\alpha^{+}-\zeta^{+}\right| & \geq\left|\alpha^{+}-\frac{p}{q}\right|-\left|\frac{p}{q}-\zeta^{+}\right| \geq \frac{e^{-M_{n} q}}{2 q^{2}}-2 \frac{e^{-2 M_{0} q}}{q^{2}} \\
& \geq \frac{e^{-M_{n} q}}{2 q^{2}}\left[1-4 e^{-M_{0} q}\right] \geq \frac{e^{-M_{n} q}}{4 q^{2}} \geq \frac{e^{-M_{n} k}}{4 k^{2}}
\end{aligned}
$$

If $n \in N$ is fixed and $k \geq \frac{\log 8}{M_{0}}$, the following decomposition shall be useful $\mathcal{Q}^{*}=\mathcal{Q}_{1}^{*}(k, n) \cup \mathcal{Q}_{0}^{*}(k, n)$ where

$$
\mathcal{Q}_{1}^{*}(k, n):=\left\{r \in \mathcal{Q}^{*}: q(k, r) \geq Q_{n}\right\}, \quad \mathcal{Q}_{0}^{*}(k, n):=\left\{r \in \mathcal{Q}^{*}: q(k, r)<Q_{n}\right\}
$$

Let $\kappa \in] 0,1$ [ be fixed and let us carry over the construction of subsection 3.1: if $V_{r}^{*}$ is a connected component $A^{*}$ and $\Delta_{r}^{*}$ is the $\kappa$-diamond over $V_{r}^{*}$ we call

$$
\Omega^{*}:=\bigcup_{r \in \mathcal{Q}^{*}} \Delta_{r}^{*}
$$

it is then easy to check that $\Omega^{*}$ is an open neighbourhood of $\mathbb{Q} / \mathbb{Z}$.

The closed set $K^{*}:=(\mathbb{C} / \mathbb{Z}) \backslash \Omega^{*}$ is connected and does not contain any rational number.

The set $C^{*}:=\exp ^{\#}\left(K^{*}\right) \cup\{\infty\}$ will be the domain on which the conjugation will be Whitney smooth. 
4.2. Proof of the regularity. - As before, let $H_{k}:=\max _{\lambda \in C_{3 M_{0}}}\left|h_{k}(\lambda)\right|$, and let $\rho_{0}=e^{-\left(3 M_{0}+b_{1}\right)}$ be the radius of convergence of $\sum H_{k} z^{k}$. We are now able to show that $h \in \mathcal{C}_{\text {hol }}^{\infty}\left(C^{*} ; \mathcal{H}^{\infty}\left(\mathbb{D}_{\rho}\right)\right)$ for every $\left.\rho \in\right] 0, e^{-1} \rho_{0}[$.

THEOREM 2. - For all $n, m \in \mathbb{N}$ there exist constants $L_{m}, \Lambda_{m, n}$ satisfying

1. $\max _{\lambda \in C^{*}}\left|h_{k}^{(m)}(\lambda)\right| \leq L_{m} k^{2 m+2} H_{k} e^{k}, \quad \forall \lambda \in C^{*} ;$

2. $\sup _{\lambda_{0}, \lambda_{1} \in C^{*}} \frac{\left|R_{n+1}\left(h_{k}^{(m)}, \lambda_{1}, \lambda_{0}\right)\right|}{\left|\lambda_{1}-\lambda_{0}\right|^{n}} \mid \leq \Lambda_{m, n} k^{2 m+2 n+2} H_{k} e^{k}, \quad \forall \lambda \in C^{*}$, where

$$
R_{n+1}\left(f, \lambda_{1}, \lambda_{0}\right):=f\left(\lambda_{1}\right)-\sum_{j=0}^{n} \frac{f^{(j)}}{j !}\left(\lambda_{0}\right)\left(\lambda_{1}-\lambda_{0}\right)^{j}
$$

is the Taylor remainder of order $n+1$.

Before plunging into the proof let us remark that the theorem implies $h \in$ $\mathcal{C}_{\text {hol }}^{\infty}\left(C^{*} ; \mathcal{H}^{\infty}\left(\mathbb{D}_{\rho}\right)\right)$ as soon as $\left.\rho \in\right] 0, e^{-1} \rho_{0}[$; indeed, (1) and (2) imply that the series $\sum_{k=0}^{+\infty} h_{k}(\lambda) z^{k}$ is normally convergent in $\mathcal{C}_{h o l}^{m}\left(C^{*} ; \mathcal{H}^{\infty}\left(\mathbb{D}_{\rho}\right)\right)$ for all $m \in \mathbb{N}$.

Proof. - We have already seen that

$$
h_{k}^{(m)}(\lambda)=\sum_{s \in \mathcal{Q}_{3 M_{0}, k}} \frac{m !}{2 \pi i} \int_{\partial D_{s}} \frac{h_{k}(\zeta)}{(\zeta-\lambda)^{m+1}} d \zeta
$$

hence

$$
\left|h_{k}^{(m)}(\lambda)\right| \leq H_{k} \frac{m !}{2 \pi} \sum_{s \in \mathcal{Q}_{3 M_{0}, k}} \int_{\partial D_{s}} d\left(\zeta, C^{*}\right)^{-m-1}|d \zeta| .
$$

Moreover we can write down an explicit expression for the Taylor remainder of $\phi_{\zeta}(\lambda):=\frac{1}{(\zeta-\lambda)^{m+1}}$ :

$$
R_{n}\left(\phi_{\zeta}, \lambda_{1}, \lambda_{0}\right)=\sum_{k=0}^{m}\left(\begin{array}{c}
k+n-1 \\
k
\end{array}\right)\left(\zeta-\lambda_{1}\right)^{k-m-1}\left(\zeta-\lambda_{0}\right)^{-n-k}\left(\lambda_{1}-\lambda_{0}\right)^{n},
$$

and we shall use the form

$$
\begin{aligned}
R_{n+1}\left(\phi_{\zeta}, \lambda_{1}, \lambda_{0}\right)= & R_{n}\left(\phi_{\zeta}, \lambda_{1}, \lambda_{0}\right)-\frac{\phi_{\zeta}^{(n)}\left(\lambda_{0}\right)}{n !}\left(\lambda_{1}-\lambda_{0}\right)^{n} \\
= & \left(\lambda_{1}-\lambda_{0}\right)^{n}\left[\sum_{k=0}^{m}\left(\begin{array}{c}
k+n-1 \\
k
\end{array}\right)\left(\zeta-\lambda_{1}\right)^{k-m-1}\left(\zeta-\lambda_{0}\right)^{-n-k}\right. \\
& \left.-\left(\begin{array}{c}
m+n \\
m
\end{array}\right)\left(\zeta-\lambda_{0}\right)^{-m-n-1}\right]
\end{aligned}
$$


On the other hand,

$$
R_{n+1}\left(h_{k}^{(m)}, \lambda_{1}, \lambda_{0}\right)=\sum_{s \in \mathcal{Q}_{3 M_{0}, k}} \frac{m !}{2 \pi i} \int_{\partial D_{s}} h_{k}(\zeta) R_{n+1}\left(\frac{1}{(\zeta-\lambda)^{m+1}}, \lambda_{1}, \lambda_{0}\right) d \zeta
$$

Using the bound $\left|\zeta-\lambda_{i}\right| \geq d\left(\zeta, C^{*}\right)(i=0,1)$ and the identity $\sum_{k=0}^{m}\left(\begin{array}{c}k+n-1 \\ k\end{array}\right)=$ $\left(\begin{array}{c}m+n \\ m\end{array}\right)$ we get

$$
\frac{\left|R_{n+1}\left(h_{k}^{(m)}, \lambda_{1}, \lambda_{0}\right)\right|}{\left|\lambda_{1}-\lambda_{0}\right|^{n}} \leq 2 H_{k} \frac{m !}{2 \pi}\left(\begin{array}{c}
m+n \\
m
\end{array}\right) \sum_{s \in \mathcal{Q}_{3 M_{0}, k}} \int_{\partial D_{s}} d\left(\zeta, C^{*}\right)^{-m-n-1}|d \zeta| .
$$

By virtue of (27) and (28) the proof of the theorem boils down to the following lemma

Lemma 12. - We have

$$
\mathcal{S}(k, \ell):=\sum_{s \in \mathcal{Q}_{3 M_{0}, k}} \int_{\partial D_{s}} d\left(\zeta, C^{*}\right)^{-\ell}|d \zeta| \leq C(\ell)\left(1+k^{2 \ell}\right) e^{k} .
$$

Proof of the Lemma. - First we split the sum as follows

$$
\mathcal{S}(k, \ell)=\sum_{r \in \mathcal{Q}^{*}} \sum_{s \in V_{r}^{*} \cap \mathcal{Q}_{3 M_{0}, k}} \int_{\partial D_{s}} d\left(\zeta, C^{*}\right)^{-\ell}|d \zeta| .
$$

Again

$$
d\left(\partial D_{s}, C^{*}\right) \geq \eta^{-1} d\left(\partial \Delta_{s}, K_{d}^{*}\right) \geq \eta_{1}^{-1} d\left(\partial V_{s}, K^{*}\right) .
$$

with $\eta_{1}:=\eta\left(1+k^{-2}\right)^{1 / 2}$. Since

On the other hand, if $s \in V_{r}^{*} \cap \mathcal{Q}_{3 M_{0}, k}$ then $d\left(\partial V_{s}, K^{*}\right) \geq d\left(\partial V_{s(r, k)}, K^{*}\right)$.

$\int_{\partial D_{s}} d\left(\zeta, C^{*}\right)^{-\ell}|d \zeta| \leq \eta_{1}^{\ell} d\left(\partial V_{s(r, k)}, K^{*}\right)^{-\ell} \int_{\partial D_{s}}|d \zeta| \leq 2 \eta_{1}^{\ell+1} d\left(\partial V_{s(r, k)}, K^{*}\right)^{-\ell}\left|V_{s}\right|$

and

$$
\sum_{s \in V_{r}^{*} \cap \mathcal{Q}_{3 M_{0}, k}}\left|V_{s}\right| \leq\left|V_{r}^{*}\right|
$$

the following estimate holds

$$
\begin{aligned}
\sum_{s \in V_{r}^{*} \cap \mathcal{Q}_{3 M_{0}, k}} \int_{\partial D_{s}} d\left(\zeta, C^{*}\right)^{-\ell}|d \zeta| & \leq 2 \eta_{1}^{\ell+1} d\left(\partial V_{s(r, k)}, K^{*}\right)^{-\ell} \sum_{s \in V_{r}^{*} \cap \mathcal{Q}_{3 M_{0}, k}}\left|V_{s}\right| \\
& \leq 2 \eta_{1}^{\ell+1} d\left(\partial V_{s(r, k)}, K^{*}\right)^{-\ell}\left|V_{r}^{*}\right| .
\end{aligned}
$$

TOME $136-2008-\mathrm{N}^{\mathrm{O}} 4$ 
Now we fix $n$ big enough so that it satisfies $M_{n} \cdot \ell<1$ and $Q(n) \geq \frac{\log 8}{M_{0}}$. We get the following estimates

$$
d\left(\partial V_{s(r, k)}, K^{*}\right) \geq \begin{cases}\nu_{0} \frac{e^{-M_{0} Q_{n}}}{Q_{n}^{2}} & \text { if } r \in \mathcal{Q}_{0}^{*}(k, n), \\ \frac{e^{-M_{n} k}}{k^{2}} & \text { if } r \in \mathcal{Q}_{1}^{*}(k, n) .\end{cases}
$$

Since $\mathcal{Q}^{*}=\mathcal{Q}_{0}^{*}(k, n) \cup \mathcal{Q}_{1}^{*}(k, n)$ (see notation in the previous section) we can split the double sum on the right hand side of (29) and get the estimate

$$
\begin{aligned}
\mathcal{S}(k, \ell) \leq & 2 \eta_{1}^{\ell+1}\left[\nu_{0}^{-\ell} Q_{n}^{2} e^{M_{0} \ell Q_{n}}\left(\sum_{r \in \mathcal{Q}_{0}^{*}(k, n)}\left|V_{r}\right|\right)\right. \\
& \left.+4 k^{2 \ell} e^{M_{n} \ell k}\left(\sum_{r \in \mathcal{Q}_{1}^{*}(k, n)}\left|V_{r}\right|\right)\right] \\
\leq & C_{0}(n, \ell)+C_{1}(\ell) k^{2 \ell} e^{k} .
\end{aligned}
$$

This ends the proof of the lemma.

\section{Gevrey regularity on Diophantine points}

Let $\mathcal{H}$ be a Banach space and let $\mathcal{G}_{\tau_{0}}\left(\lambda_{0}, \mathcal{H}\right)$ be the vector space of all $\mathcal{H}$ valued functions $h$ for which there exist two (disjoint) open disks $\Delta^{ \pm}$tangent to $\mathbb{S}^{1}$ at $\lambda_{0}$, a formal series $\sum_{k \geq 0} c_{k} \Lambda^{k} \in \mathcal{H}[[\Lambda]]$ and positive numbers $b_{1}$ and $b_{2}$ such that the function $h$ is holomorphic in $\Delta^{+} \cup \Delta^{-}$and it has a Gevrey- $\tau_{0}$ asymptotic expansion at $\lambda_{0}$, i.e.

$\forall N \geq 0, \forall \lambda \in \Delta^{+} \cup \Delta^{-},\left\|h(q)-\sum_{k=0}^{N-1} c_{k}\left(\lambda-\lambda_{0}\right)^{k}\right\| \leq b_{1} b_{2}^{N} \Gamma\left(1+N \tau_{0}\right)\left|\lambda-\lambda_{0}\right|^{N}$,

where $\Gamma$ is Euler's Gamma function.

In the following we shall slightly change our notation and set $H(\lambda, z)=$ $H_{\lambda}(z)$. It should be clear that Theorem B is a straightforward consequence of the following proposition:

Proposition 5.1. - Let $\alpha_{0} \in D C\left(\tau_{0}, \gamma_{0}\right)$ be a fixed diophantine number and let $\lambda_{0}=e^{2 \pi i \alpha_{0}}$. Let $\Delta^{ \pm}$be a pair of disks which are tangent to $\lambda_{0}, \Delta^{+} \subset \mathbb{D}$, $\Delta^{-} \subset \mathbb{C} \backslash \mathbb{D}$. Moreover let $\rho \in\left(0, e^{-1} \rho_{0}\right)$ and $\mathcal{H}=\mathcal{H}^{\infty}\left(\mathbb{D}_{\rho}\right)$. Then

(a) $H \in \mathcal{C}_{\text {hol }}^{\infty}\left(\overline{\Delta^{+} \cup \Delta^{-}}, \mathcal{H}^{\infty}\left(\mathbb{D}_{\rho}\right)\right.$ ) (hence $H$ is holomorphic on $\Delta^{+} \cup \Delta^{-}$). 
(b) There are constants $b_{1}, b_{2}$ such that

$$
\left\|H-\sum_{j=0}^{N-1} \frac{1}{j !} \partial_{\lambda}^{j} H\left(\lambda_{0}, \cdot\right)\left(\lambda-\lambda_{0}\right)^{j}\right\|_{\mathcal{H}} \leq b_{1} b_{2}^{N} \Gamma\left(1+\tau_{0} N\right)\left|\lambda-\lambda_{0}\right|^{N}
$$

for all $N \in \mathbb{N}, \lambda \in \Delta^{ \pm}$.

5.1. More Cantor sets related with the diophantine condition. - In order to prove Proposition 5.1 we shall use just a few definitions and results from the paper [10] ${ }^{(7)}$. For $\tau \geq 2, \gamma \in(0,1)$ let

$$
K_{\gamma, \tau}:=\left\{x \in(\mathbb{R} \backslash \mathbb{Q}) / \mathbb{Z}: \forall k \geq 0 q_{k+1} \leq \gamma^{-1} q_{k}^{\tau-1}\right\} ;
$$

It is readily seen that $K_{\gamma, \tau}$ is a compact subset of $\mathbb{R} / \mathbb{Z}$ and

$$
D(\gamma, \tau) \subset K_{\gamma, \tau} \subset D\left(\frac{\gamma}{2}, \tau\right)
$$

(see also A3.2 in [10]). The proof of the following proposition can be found in [10] (Proposition 2.2).

Proposition 5.2. - $\quad$ 1. Each connected component $] \xi^{-}, \xi^{+}\left[\right.$of $(\mathbb{R} / \mathbb{Z}) \backslash K_{\gamma, \tau}$ contains a unique rational number $p / q$ which is a convergent of both endpoints $\xi^{ \pm}$. Such convergent $p / q$ is the pseudocenter of the component ]$\xi^{-}, \xi^{+}\left[\right.$. We shall denote $\mathcal{Q}_{\gamma, \tau}$ the set of pseudocenters of all connected components of $(\mathbb{R} / \mathbb{Z}) \backslash K_{\gamma, \tau}$.

2. $\frac{\gamma}{2} q^{-\tau} \leq\left|\xi^{ \pm}-\frac{p}{q}\right| \leq 2 \gamma q^{-\tau}$.

Let us remark that, if $\alpha \in K_{\gamma, \tau}$ then $q_{k+1}=a_{k+1} q_{k}+q_{k-1} \leq \gamma^{-1} q^{\tau-1}$ and hence $a_{k+1} \leq \gamma^{-1} q^{\tau-2}$. From this information we get not only an a priori estimates for $\mathcal{B}(\alpha)$ but also

$$
\sum_{k=n}^{+\infty} \frac{\log a_{k+1}}{q_{k}} \leq\left(\log \gamma^{-1}\right) \sum_{k=n}^{+\infty} \frac{1}{q_{k}}+(\tau-2) \sum_{k=n}^{+\infty} \frac{\log q_{k}}{q_{k}} .
$$

This is interesting because, since $q_{k} \geq F_{k}$, we get that

$$
\sum_{k=n}^{+\infty} \frac{1}{q_{k}} \leq \sum_{k=n}^{+\infty} \frac{1}{F_{k}}, \quad \sum_{k=n}^{+\infty} \frac{\log q_{k}}{q_{k}} \leq \sum_{k=n}^{+\infty} \frac{\log F_{k}}{F_{k}}
$$

therefore setting

$$
M_{n}:=\left(\log \gamma^{-1}\right) \sum_{k=n}^{+\infty} \frac{1}{F_{k}}+(\tau-2) \sum_{k=n}^{+\infty} \frac{\log F_{k}}{F_{k}}
$$

(7) Let us point out that we do not stick to the notation used in [10]: in particular we shall call " $K_{\gamma, \tau}$ " the set that in [10] is called $C_{\psi_{\gamma, \tau}}$. 
we see that $K_{\gamma, \tau} \subset K_{\left(M_{n}\right)}^{*}:=\left\{x \in \mathbb{R} / \mathbb{Z}: \sum_{k=n}^{+\infty} \frac{\log a_{k+1}}{q_{k}} \leq M_{n}\right\}$. For $s \in \mathcal{Q}_{\gamma, \tau}$ let $V_{s}$ be the corresponding connected component of $(\mathbb{R} / \mathbb{Z}) \backslash K_{\gamma, \tau}, \Delta_{s}$ be the diamond over $V_{s}, D_{s}:=\exp ^{\#}\left(\Delta_{s}\right)$ and $C_{\gamma, \tau}=\mathbb{C} \backslash \cup_{s \in \mathcal{Q}_{\gamma, \tau}} D_{s}$. We then have that $C_{\gamma, \tau}$ is contained in the set of $\mathcal{C}^{\infty}$-regularity $C_{\left(M_{n}\right)}^{*}$.

The following geometrical lemma is useful to settle both (a) and (b) of Proposition 5.1.

LEMMA 13. - Let $\tau_{0} \geq 2, \alpha_{0} \in D C\left(\tau_{0}, \gamma_{0}\right), \lambda_{0}=e^{2 \pi i \alpha_{0}}$. Let $Q$ be a closed set satisfying

(i) $Q \cap \mathbb{S}^{1}=\left\{\lambda_{0}\right\}$;

(ii) there exist $\mu_{0}>0$ such that $\operatorname{dist}\left(\lambda, \mathbb{S}^{1}\right) \geq \mu_{0}\left|\lambda-\lambda_{0}\right|^{2} \forall \lambda \in Q$.

Then for any fixed $\tau>2 \tau_{0}$ there exists $\gamma \in\left(0, \gamma_{0}\right)$ such that, defining $\left(M_{n}\right)$ as in (33) so that $C_{\gamma, \tau} \subset C_{\left(M_{n}\right)}^{*}$,

(a') $Q \subset C_{\gamma, \tau} \subset C_{\left(M_{n}\right)}^{*}$;

$\left(\mathrm{b}^{\prime}\right)$ there exists $\mu>0$ such that $\operatorname{dist}(\zeta, Q) \geq \mu \gamma_{0}^{2} q^{-2 \tau_{0}} \quad \forall \zeta \in \partial D_{p / q},(p / q \in$ $\left.\mathcal{Q}_{\gamma, \tau}\right)$.

Proof of the Lemma. - Let us consider $Q_{0}:=Q \cap\left\{z:\left|z-\lambda_{0}\right| \leq 1 / 2\right\}$ and $Q_{1}:=Q \cap\left\{z:\left|z-\lambda_{0}\right| \geq 1 / 2\right\}$, we shall prove that (a) and (b) hold on both the closed sets $Q_{0}$ and $Q_{1}$ and hence hold on $Q$ as well. Of course, in the case of $Q_{1}$ there is no problem: since $Q_{1} \cap \mathbb{S}^{1}=\varnothing$ the points of $Q_{1}$ are bounded away from $\mathbb{S}^{1}$ and both (a) and (b) hold provided that $\gamma$ and $\mu$ are small enough. As far as $Q_{0}$ is concerned, we observe that the logarithm is well defined on $\left\{z:\left|z-\lambda_{0}\right| \leq 1 / 2\right\}$ and it has a bounded distorsion property, therefore wecan check both (a) and (b) for $Q_{0}$ just proving the following statement:

If $\mu_{0}^{\prime}>0$ and $Q^{\prime}:=\left\{\alpha: \Im \alpha \geq \mu_{0}^{\prime}\left|z-\alpha_{0}\right|^{2},|\Re \alpha| \leq \pi / 6\right\}$ there exist $\gamma, \mu^{\prime}>0$ such that $\operatorname{dist}\left(\xi, Q^{\prime}\right) \geq \mu^{\prime} \gamma_{0}^{2} q^{-2 \tau_{0}} \quad \forall \xi \in \partial \Delta_{p / q}, p / q \in \mathcal{Q}_{\gamma, \tau}$.

This is readily checked because, if $\xi \in \partial \Delta_{p / q}$ is fixed and $\alpha(\xi)$ is the nearest point in $Q^{\prime}$ we have that $|\alpha(\xi)-\xi| \geq|\alpha(\xi)-p / q|-|\xi-p / q|$; on the other hand we have that

$$
|\alpha(\xi)-p / q| \geq C|\Im \alpha| \geq C \mu_{0}^{\prime}|\alpha-p / q|^{2} \geq\left(C \mu_{0}^{\prime} \gamma_{0}^{2}\right) q^{-2 \tau_{0}}
$$

while

$$
|\xi-p / q| \leq 2 \frac{\kappa}{\sqrt{1+\kappa^{2}}} \gamma q^{-\tau}=O\left(q^{-\tau}\right)
$$

Let us point out that by means of the same argument used in the proof of the last lemma we also get

$$
\left|\lambda_{0}-\zeta\right| \geq \mu \gamma_{0} q^{-\tau_{0}} \quad \forall \zeta \in \partial D_{p / q},\left(p / q \in \mathcal{Q}_{\gamma, \tau}\right) .
$$


5.2. Proof of Gevrey regularity. - By $\left(\mathrm{a}^{\prime}\right)$ of Lemma 13 we get that, if $\tau>2 \tau_{0}$, it is possible to choose $\gamma$ such that $\overline{\Delta^{+} \cup \Delta^{-}} \subset C_{\gamma, \tau} \subset C_{\left(M_{n}\right)}^{*}$ hence $H$ is $\mathcal{C}_{\text {hol }}^{\infty}\left(\overline{\Delta^{+} \cup \Delta^{-}}, \mathcal{H}^{\infty}\left(\mathbb{D}_{\rho}\right)\right)$ in particular $H$ admits a Taylor expansion at $\lambda_{0}$. We shall set

$$
\mathbf{R}_{N}\left(H, \lambda, \lambda_{0}\right):=H(\lambda, \cdot)-\sum_{j=0}^{N-1} \frac{1}{j !} \partial_{\lambda}^{j} H\left(\lambda_{0}, \cdot\right)\left(\lambda-\lambda_{0}\right)^{j}
$$

so that $\mathbf{R}_{N}\left(H, \cdot, \lambda_{0}\right): \Delta^{+} \cup \Delta^{-} \rightarrow \mathcal{H}^{\infty}\left(\mathbb{D}_{\rho}\right)$. We point out that, setting

$$
R_{N}\left(h_{k}, \lambda, \lambda_{0}\right):=h_{k}(\lambda)-\sum_{j=0}^{N-1} \frac{1}{j !} h_{k}^{(j)}\left(\lambda_{0}\right)\left(\lambda-\lambda_{0}\right)^{j}
$$

we can write

$$
\mathbf{R}_{N}\left(H, \lambda, \lambda_{0}\right)(z)=\sum_{k=1}^{+\infty} R_{N}\left(h_{k}, \lambda, \lambda_{0}\right) z^{k}
$$

Let us recall that, by Cauchy formula,

$$
R_{N}\left(h_{k}, \lambda, \lambda_{0}\right)=\sum_{s \in \mathcal{Q}_{\gamma, \tau, k}} \frac{1}{2 \pi i} \int_{\partial D_{s}} \frac{h_{k}(\zeta)}{\left(\zeta-\lambda_{0}\right)^{N}(\zeta-\lambda)}\left(\lambda-\lambda_{0}\right)^{N} d \zeta
$$

where $\mathcal{Q}_{\gamma, \tau, k}=\mathcal{Q}_{\gamma, \tau} \cap \mathbb{Q}_{k}$. Moreover, if $\lambda \in \Delta^{+} \cup \Delta^{-}$and $\zeta \in \partial D_{s}$ with $s=p / q \in \mathcal{Q}_{\gamma, \tau, k}$ then

$$
\left|h_{k}(\zeta)\right| \leq H_{k}, \quad\left|\zeta-\lambda_{0}\right| \geq \mu \gamma_{0}|q|^{-\tau_{0}} \quad \text { and } \quad|\zeta-\lambda| \geq \mu \gamma_{0}^{2}|q|^{-2 \tau_{0}}
$$

so we get the bound

$$
\left|R_{N}\left(h_{k}, \lambda, \lambda_{0}\right)\right| \leq\left(\mu \gamma_{0}\right)^{-N-2} k^{(N+2) \tau_{0}} H_{k} \frac{1}{2 \pi} \sum_{s \in \mathcal{Q}_{3 M_{0}, k}} \int_{\partial D_{s}}|d \zeta|\left|\lambda-\lambda_{0}\right|^{N}
$$

The sum on the right hand side of the last formula is bounded by a constant (independent from $k$ and $N$ ) moreover, since we have chosen $\rho<\rho_{0}$, we can fix $C_{1}$ such that $k^{2 \tau_{0}} H_{k} \rho^{k} \leq C_{1} e^{-k}$. Thus, summing up on $k$, we get

$$
\left\|\mathbf{R}_{N}\left(H, \lambda, \lambda_{0}\right)\right\| \leq \sum_{k=1}^{+\infty}\left|R_{n}\left(h_{k}, \lambda, \lambda_{0}\right)\right| \rho^{k} \leq C_{2}\left(\mu \gamma_{0}\right)^{-N}\left(\sum_{k=0}^{+\infty} k^{N \tau_{0}} e^{-k}\right)\left|\lambda-\lambda_{0}\right|^{N}
$$

where $C_{2}$ is a suitable constant. Since

$$
\sum_{k=0}^{+\infty} k^{\beta} e^{-k} \leq \int_{0}^{+\infty} t^{\beta} e^{-t} d t+(\beta / e)^{\beta} \leq 2 \Gamma(1+\beta)
$$

the thesis follows. 


\section{Appendix A}

\section{Arithmetical tools and Brjuno series}

Let us recall some notation and elementary facts about classical continued fractions. We refer the reader to [6] and [8] for more details. Continued fractions are obtained by coding the orbits of real numbers under the iteration of the Gauss map $\mathcal{G}:(0,1) \mapsto[0,1]$ defined by $\mathcal{G}(x)=\left\{x^{-1}\right\}=x^{-1}-\left[x^{-1}\right]$ where $[x]$ and $\{x\}$ respectively denote the integer and the fractional part of $x$. This map is piecewise analytic with inverse branches $T_{n}(x)=\frac{1}{n+x}, T_{n}=\mathcal{G}^{-1}$ on the interval $\left(\frac{1}{n+1}, \frac{1}{n}\right)$. Given $x \in \mathbb{R} \backslash \mathbb{Q}$ we set $x_{0}=x-[x], a_{0}=[x]$, then one obviously has $x=a_{0}+x_{0}$. We now define inductively for all $n \geq 0$ $x_{n+1}=\mathcal{G}\left(x_{n}\right), a_{n+1}=\left[x_{n}^{-1}\right] \geq 1$, thus $x_{n}=T_{a_{n+1}}\left(x_{n+1}\right)$. Therefore we have

$$
x=a_{0}+T_{a_{1}}\left(x_{1}\right)=\cdots=a_{0}+T_{a_{1}} \circ \ldots \circ T_{a_{n}}\left(x_{n}\right)=a_{0}+\frac{1}{a_{1}+\frac{1}{a_{2}+\ddots+\frac{1}{a_{n}+x_{n}}}} .
$$

We will use the short notation $x=\left[a_{0}, a_{1}, \ldots, a_{n}, \ldots\right]$ for the infinite fraction. The nth-convergent is then the rational number corresponding to the finite fraction $\frac{p_{n}}{q_{n}}=\left[a_{0}, a_{1}, \ldots, a_{n}\right]$.

The numerators $p_{n}$ and denominators $q_{n}$ are recursively determined for all $n \geq 0$ by

$$
p_{n}=a_{n} p_{n-1}+p_{n-2}, \quad q_{n}=a_{n} q_{n-1}+q_{n-2},
$$

with the initial conditions $p_{-1}=q_{-2}=1, p_{-2}=q_{-1}=0$. Note that $q_{n} p_{n-1}-$ $p_{n} q_{n-1}=(-1)^{n}$.

For all $n \geq 0$ one also has

$$
x=\frac{p_{n}+p_{n-1} x_{n}}{q_{n}+q_{n-1} x_{n}}, \quad x_{n}=-\frac{q_{n} x-p_{n}}{q_{n-1} x-p_{n-1}},
$$

thus for all $k \geq 0$ and for all $x \in \mathbb{R} \backslash \mathbb{Q}$ one has $\frac{p_{2 k}}{q_{2 k}}<x<\frac{p_{2 k+1}}{q_{2 k+1}}$.

It is not difficult to show that for all $x \in \mathbb{R} \backslash \mathbb{Q}$ and for all $n \geq 1$ one has $q_{n} \geq \frac{1}{2} \phi_{0}^{1-n}$, with $\phi_{0}=\frac{\sqrt{5}-1}{2}$. This implies that the series $\sum_{k=0}^{\infty} \frac{\log q_{k}}{q_{k}}$ and $\sum_{k=0}^{\infty} \frac{1}{q_{k}}$ are always convergent and that their sum is uniformly bounded.

For all integers $k \geq 1$, the iteration of the Gauss map $k$ times leads to the following partition of $(0,1) ; \sqcup_{a_{1}, \ldots, a_{k}} I\left(0, a_{1}, \ldots, a_{k}\right)$, where $a_{i} \in \mathbb{N}, i=1, \ldots, k$, and

$$
\begin{aligned}
I\left(0, a_{1}, \ldots, a_{2 k}\right) & =\left(\frac{p_{2 k}}{q_{2 k}}, \frac{p_{2 k}+p_{2 k-1}}{q_{2 k}+q_{2 k-1}}\right) \\
I\left(0, a_{1}, \ldots, a_{2 k+1}\right) & =\left(\frac{p_{2 k+1}+p_{2 k}}{q_{2 k+1}+q_{2 k}}, \frac{p_{2 k+1}}{q_{2 k+1}}\right) .
\end{aligned}
$$


These are the intervals corresponding to the branches of $\mathcal{G}^{k}$ : they are determined by the fact that all points $x \in I\left(0, a_{1}, \ldots, a_{k}\right)$ have the first $k+1$ partial quotients exactly equal to $\left\{0, a_{1}, \ldots, a_{k}\right\}$. Thus

$$
I\left(0, a_{1}, \ldots, a_{k}\right)=\left\{x \in(0,1) \mid x=\frac{p_{k}+p_{k-1} y}{q_{k}+q_{k-1} y}, y \in(0,1)\right\} .
$$

Note that $\frac{d x}{d y}=\frac{(-1)^{k}}{\left(q_{k}+q_{k-1} y\right)^{2}}$ is positive (negative) if $k$ is even (odd). It is immediate to check that any rational number $p / q \in(0,1),(p, q)=1$, is the endpoint of exactly two branches of the iterated Gauss map. Indeed $p / q$ can be written as $p / q=\left[0, \bar{a}_{1}, \ldots, \bar{a}_{k}\right]$ with $k \geq 1$ and $\bar{a}_{k} \geq 2$ in a unique way and it is the left (right) endpoint of $I\left(0, \bar{a}_{1}, \ldots, \bar{a}_{k}\right)$ and the right (left) endpoint of $I\left(0, \bar{a}_{1}, \ldots, \bar{a}_{k}-1,1\right)$ if $k$ is even (odd).

The cylinders $I\left(a_{0}, \ldots, a_{N}\right)=a_{0}+I\left(0, a_{1}, \ldots, a_{N}\right)$ form a partition of the whole real line as $a_{0}$ varies in $\mathbb{Z}$ and $\left(a_{1}, \ldots, a_{n}\right) \in \mathbb{N}^{n}$.

\section{Appendix B}

\section{$\mathcal{C}^{1}$-holomorphic and $\mathcal{C}^{\infty}$-holomorphic functions}

Let $(B,\|\|)$ be a complex Banach space. In this appendix we recall the definition of $\mathcal{C}^{1}$-holomorphic and $\mathcal{C}^{\infty}$-holomorphic functions as they are given respectively in [7] and [13]. We follow quite closely Section 2 of [10] to which we refer for a more detailed discussion.

Let $C$ be a compact subset of $\mathbb{C}$ or of $\mathbb{P}^{1} \mathbb{C}$. If $C \subset \mathbb{C}$, a continuous function $f: C \rightarrow B$ is said to be $\mathcal{C}^{1}$-holomorphic if there exists a continuous map $f^{(1)}: C \rightarrow B$ such that

$$
\begin{aligned}
\forall \lambda \in C, \forall \varepsilon>0, \exists \delta & >0 / \forall \lambda_{1}, \lambda_{2} \in C \\
& \left|\lambda_{1}-\lambda\right|<\delta,\left|\lambda_{2}-\lambda\right|<\delta \\
& \Rightarrow\left\|f\left(\lambda_{2}\right)-f\left(\lambda_{1}\right)-f^{(1)}\left(\lambda_{1}\right)\left(\lambda_{2}-\lambda_{1}\right)\right\| \leq \varepsilon\left|\lambda_{1}-\lambda_{2}\right|
\end{aligned}
$$

This definition extends in an obvious way to the case $C \subset \mathbb{P}^{1} \mathbb{C}$ by means of the standard complex coordinates charts.

The above definition makes use of the generalization of the notion of smoothness of a function to a closed set due to Whitney [16]. Notice however that $f^{(1)}$ is a complex derivative: $\bar{\partial} f=0, \partial f=f^{(1)}$ and $f$ is holomorphic in the interior of $C$.

TOME $136-2008-\mathrm{N}^{\mathrm{O}} 4$ 
The space $\mathcal{C}_{\text {hol }}^{1}(C, B)$ becomes a Banach space by taking as norm

$$
\begin{aligned}
& \|f\|=\max \left(\sup _{\lambda \in C}\|f(\lambda)\|, \sup _{\lambda \in C}\left\|f^{(1)}(\lambda)\right\|,\right. \\
& \left.\sup _{\lambda_{1}, \lambda_{2} \in C, \lambda_{1} \neq \lambda_{2}} \frac{\left\|f\left(\lambda_{2}\right)-f\left(\lambda_{1}\right)-f^{(1)}\left(\lambda_{1}\right)\left(\lambda_{2}-\lambda_{1}\right)\right\|}{\left|\lambda_{1}-\lambda_{2}\right|}\right)
\end{aligned}
$$

Let $\mathcal{R}(C, B)$ denote the uniform algebra of continuous functions from $C$ to $B$ which are uniformly approximated by rational functions with all the poles outside $C$. A very important property of the space $\mathcal{C}_{h o l}^{1}(C, B)$ is that it is a linear subspace of $\mathcal{R}(C, B)$. This fact allows to prove that functions in $\mathcal{C}_{\text {hol }}^{1}(C, B)$ share some of the properties of holomorphic functions. If $\left(U_{\ell}\right)_{\ell \geq 1}$ denote the connected components of $\mathbb{P}^{1} \mathbb{C} \backslash C$, assuming that each $\partial U_{\ell}$ is a piecewise smooth Jordan curve and $\sum_{\ell \geq 1}$ length $\left(\partial U_{\ell}\right)<+\infty$, Cauchy's theorem holds:

$$
\sum_{\ell=1}^{\infty} \int_{\partial U_{\ell}} f(\lambda) d \lambda=0
$$

This is very easy to see: since $f \in \mathcal{R}(C, B)$, one can approximate $f$ by a sequence $\left(r_{k}\right)_{k \in N}$ of $B$-valued rational functions with poles off $C$. Cauchy's theorem applies to these rational functions and one can pass to the limit because the convergence is uniform. Moreover, at all points $\lambda \in C$ such that

$$
\sum_{\ell=1}^{\infty} \int_{\partial U_{\ell}} \frac{|d \zeta|}{|\zeta-\lambda|}<+\infty
$$

Cauchy's formula also holds:

$$
f(\lambda)=\frac{1}{2 \pi i} \sum_{\ell=1}^{\infty} \int_{\partial U_{\ell}} \frac{f(\zeta)}{\zeta-\lambda} d \zeta .
$$

One can also define higher order derivatives by means of Cauchy's formula, but in order to do so one needs further assumptions on $\lambda$ (namely $\sum_{\ell=1}^{\infty} \int_{\partial U_{\ell}} \frac{|d \zeta|}{|\zeta-\lambda|^{n+1}}<+\infty$ to obtain a derivative of order $n$ ).

A function $f: C \rightarrow B$ is said to be $\mathcal{C}^{\infty}$-holomorphic if there exist an infinite sequence of continuous functions $\left(f^{(n)}\right)_{n \in \mathbb{N}}: C \rightarrow B$ (the " $n$-th complex derivative of $\left.f^{\prime \prime}\right)$ such that $f^{(0)}=f$ and, for all $n, m \geq 0$, the function $R^{(n, m)}$ defined by

$$
R^{(n, m)}\left(\lambda_{1}, \lambda_{2}\right)=\sum_{h=0}^{m} \frac{f^{(n+h)}\left(\lambda_{1}\right)}{h !}\left(\lambda_{2}-\lambda_{1}\right)^{h}-f^{(n)}\left(\lambda_{2}\right), \quad \lambda_{1}, \lambda_{2} \in C,
$$


has the following property:

$$
\begin{aligned}
\forall \lambda \in C, \forall \varepsilon>0, \exists \delta>0 / \forall \lambda_{1}, \lambda_{2} \in C,\left|\lambda_{1}-\lambda\right|<\delta,\left|\lambda_{2}-\lambda\right|<\delta \Rightarrow \\
\left\|R^{(n, m)}\left(\lambda_{1}, \lambda_{2}\right)\right\| \leq \varepsilon\left|\lambda_{1}-\lambda_{2}\right|^{m} .
\end{aligned}
$$

The space of $\mathcal{C}^{\infty}$-holomorphic $B$-valued functions on a compact set is a Fréchet space. We stress once more that the derivatives are taken in a complex sense, thus $\bar{\partial} f^{(n)}=0$ for all $n \in \mathbb{N}$. The functions $f^{(n)}$ are some generalized "weak complex derivatives for $f$ "; clearly $f$ must be analytic in the interior of $C$ and

$$
\forall n, m \in \mathbb{N}, \quad \forall \lambda \in \operatorname{int}(C), \quad f^{(n+m)}(\lambda)=\partial^{m} f^{(n)}(\lambda)
$$

Let $\left(C_{j}\right)_{j \in \mathbb{N}}$ be a monotonic non-decreasing sequence of compact subsets of $\mathbb{P}^{1} \mathbb{C}$. The associated space of $B$-valued monogenic functions is defined to be the projective limit

$$
\mathcal{M}\left(\left(C_{j}\right), B\right)=\lim _{\longleftarrow} \mathcal{C}_{\text {hol }}^{1}\left(C_{j}, B\right) .
$$

The restrictions $\mathcal{C}_{h o l}^{1}\left(C_{j+1}, B\right) \rightarrow \mathcal{C}_{h o l}^{1}\left(C_{j}, B\right)$ are continuous linear operators between Banach spaces, thus $\mathcal{M}\left(\left(C_{j}\right), B\right)$ is a Fréchet space with seminorms $\|\cdot\|_{\mathcal{C}_{h o l}^{1}\left(C_{j}, B\right)}$.

The above definition is inspired by the work of Borel [2] (see also [7], p. 81). Borel considered the case $B=\mathbb{C}$ and wanted to extend the notions of holomorphic function and analytic continuation. Borel's idea was to allow monogenic continuation through natural boundaries of analyticity by selecting points at which the function is $\mathcal{C}_{h o l}^{1}$-holomorphic. If the function is moreover $\mathcal{C}_{h o l}^{\infty}$-holomorphic at such a point, the question of quasianalyticity may be raised: Is the function determined by its Taylor series? Such a uniqueness property could depend on the choice of the sequence $\left(C_{j}\right)$ which defines the monogenic class (and not only on the union of the $C_{j}$ 's), and the Cauchy formula could help to establish it.

Unfortunately this strong form of quasianalyticity is not true in general spaces of monogenic functions unless some rather restrictive assumptions are made (see, e.g. , [17]). However it is proved in [11] that it is quite a general property that the spaces of $\mathcal{C}^{1}$-holomorphic functions (and also of monogenic functions) which appear in linearization problems have a weaker quasianalyticity property, namely their functions cannot vanish on a set of positive 1dimensional Hausdorff measure without being identically equal to zero. This provides an example of generalized analytic continuation (for a comprehensive discussion of generalized analytic continuations other than Borel's theory see [14]).

The notion of $B$-valued monogenic function is well adapted to linear small denominator problems, as the cohomological equation considered in [10] but it 
is useless in nonlinear problems since one cannot fix a target Banach space if one wants the increasing sequence of compact sets to include all points on the unit circle verifying the Brjuno condition. Indeed the radius of convergence of the linearization is, in general, also bounded above in terms of the exponential of minus the Brjuno function as first proved by Yoccoz [18]. For this reason we introduce the notion of monogenic function with values in $\mathbb{C}\{z\}$ : suppose $\left(C_{j}\right)_{j \in \mathbb{N}}$ is a monotonic non-decreasing sequence of compact subsets of $\mathbb{P}^{1} \mathbb{C}$ and consider the increasing sequence of Banach spaces $\mathcal{H}^{\infty}\left(\mathbb{D}_{r_{j}}\right)$ associated to a monotonic non-increasing sequence of radii $r_{j} \rightarrow 0$. Consider the Banach space $B_{\ell}=$ $\cap_{j=0}^{\ell} \mathcal{C}_{h o l}^{1}\left(C_{j}, \mathcal{H}^{\infty}\left(\mathbb{D}_{r_{j}}\right)\right)$ with the norm $\|f\|_{B_{\ell}}=\max _{0 \leq j \leq \ell}\|f\|_{\mathcal{C}_{h o l}^{1}\left(C_{j}, \mathcal{H}^{\infty}\left(\mathbb{D}_{r_{j}}\right)\right)}$. Clearly the injections $i_{\ell}: B_{\ell} \hookrightarrow B_{\ell-1}$ are bounded linear operators between Banach spaces with norms $\left\|i_{\ell}\right\| \leq 1$. The projective limit of the system of Banach spaces

$$
\mathcal{M}\left(\left(C_{j}\right), \mathbb{C}\{z\}\right)=\lim _{\longleftarrow} B_{j}
$$

is the space of monogenic functions with values in the holomorphic germs $\mathbb{C}\{z\}$. It is a Fréchet space with the seminorms $\|\cdot\|_{B_{\ell}}$. Thus, as a set, $\mathcal{M}\left(\left(C_{j}\right), \mathbb{C}\{z\}\right)$ consists of all the functions which are defined in $C=\bigcup_{j \in \mathbb{N}} C_{j}$ and such that, for every $j \in \mathbb{N}$, the restriction $f_{\mid C_{j}}$ belongs to $\mathcal{C}_{h o l}^{1}\left(C_{j}, \mathcal{H}^{\infty}\left(\mathbb{D}_{r_{j}}\right)\right)$. This space, being the projective limit of the Banach spaces $B_{\ell}$, may depend on the increasing sequence $\left(C_{j}\right)$ and on the decreasing sequnce $r_{j}$ (rather than on the set $C$ only).

\section{BIBLIOGRAPHY}

[1] V. I. ARNOLD - "On the mappings of the circumference onto itself", Translations of the Amer. Math. Soc. 46 (1961), p. 213-284.

[2] E. BoREL - Leçons sur les fonctions monogènes uniformes d'une variable complexe, Gauthier-Villars, 1917.

[3] A. D. BRJuno - "Analytic form of differential equations. I, II", Trudy Moskov. Mat. Obšč. 25 (1971), p. 119-262; ibid. 26 (1972), 199-239.

[4] T. Carletti \& S. MARMi - "Linearization of analytic and non-analytic germs of diffeomorphisms of (C,0)", Bull. Soc. Math. France 128 (2000), p. $69-85$.

[5] A. M. DAVIE - "The critical function for the semistandard map", Nonlinearity 7 (1994), p. 219-229.

[6] G. H. Hardy \& E. M. WRIGHT - An introduction to the theory of numbers, fifth ed., The Clarendon Press Oxford University Press, 1979.

[7] M.-R. Herman - "Simple proofs of local conjugacy theorems for diffeomorphisms of the circle with almost every rotation number", Bol. Soc. Brasil. Mat. 16 (1985), p. 45-83. 
[8] A. Y. KhInchin - Continued fractions, The University of Chicago Press, Chicago, Ill.-London, 1964.

[9] A. N. Kolmogorov - "The general theory of dynamical systems and classical mechanics", in International Congress of Mathematicians, Amsterdam, 1954.

[10] S. MARmi \& D. SAUZIN - "Quasianalytic monogenic solutions of a cohomological equation", Mem. Amer. Math. Soc. 164 (2003), p. 83.

[11] _ "A quasianalyticity property for monogenic solutions of small divisor problems", preprint arXiv:0706.0138v1, 2007.

[12] J. Pöschel - "Integrability of Hamiltonian systems on Cantor sets", Comm. Pure Appl. Math. 35 (1982), p. 653-696.

[13] E. RISLER - "Linéarisation des perturbations holomorphes des rotations et applications", Mém. Soc. Math. Fr. (N.S.) (1999), p. 102.

[14] W. T. Ross \& H. S. SHAPIRO - Generalized analytic continuation, University Lecture Series, vol. 25, American Mathematical Society, 2002.

[15] V. Thilliez - "Quelques propriétés de quasi-analyticité", Gaz. Math. (1996), p. 49-68.

[16] H. Whitney - "Analytic extensions of differentiable functions defined in closed sets", Trans. Amer. Math. Soc. 36 (1934), p. 63-89.

[17] J. WINKLER - "A uniqueness theorem for monogenic functions", Ann. Acad. Sci. Fenn. Ser. A I Math. 18 (1993), p. 105-116.

[18] J.-C. YocCOZ - "Théorème de Siegel, nombres de Bruno et polynômes quadratiques", Astérisque (1995), p. 3-88, Petits diviseurs en dimension 1.

[19] _ "Analytic linearization of circle diffeomorphisms", in Dynamical systems and small divisors (Cetraro, 1998), Lecture Notes in Math., vol. 1784, Springer, 2002, p. 125-173. 